TOPICAL REVIEW

Human augmentation by wearable supernumerary robotic limbs: review and perspectives

To cite this article: Domenico Prattichizzo et al 2021 Prog. Biomed. Eng. 3042005

View the article online for updates and enhancements. 


\title{
Progress in Biomedical Engineering
}

\author{
TOPICAL REVIEW
}

CrossMark

RECEIVED

9 February 2021

REVISED

5 July 2021

ACCEPTED FOR PUBLICATION

31 August 2021

PUBLISHED

17 September 2021

\section{Human augmentation by wearable supernumerary robotic limbs: review and perspectives}

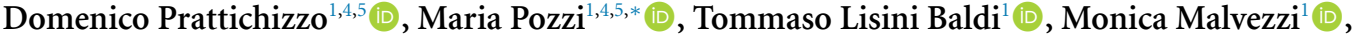 \\ Irfan Hussain $^{3}$ (D), Simone Rossi ${ }^{2}$ (D) and Gionata Salvietti ${ }^{1}$ (D) \\ 1 Department of Information Engineering and Mathematics, University of Siena, Siena, Italy \\ 2 Department of Medicine, Surgery and Neuroscience, University of Siena, Siena, Italy \\ 3 Department of Mechanical Engineering, Khalifa University, Abu Dhabi, United Arab Emirates \\ 4 Equally contributed to this work. \\ 5 Humanoids \& Human Centered Mechatronics Research Line, Istituto Italiano di Tecnologia (IIT), Genova, Italy \\ * Author to whom any correspondence should be addressed. \\ E-mail: maria.pozzi@unisi.it
}

Keywords: wearable supernumerary robotic limbs, wearable interfaces, wearable robotics, human-robot interaction, robotic manipulation

\begin{abstract}
Supernumerary robotic limbs (SRLs) are wearable robots designed to enhance the sensorimotor abilities of humans. SRLs can be used to compensate for lost functions in patients with motor deficits and, more in general, to augment the sensorimotor capabilities of humans to interact with the environment. The design and control of SRLs present several challenges. SRLs must have high levels of ergonomics and wearability and, depending on the application, they might also require enhanced robustness and strength. Supernumerary robotics differs from collaborative robotics, since SRLs are not collaborative external agents but rather act under the direct command of the operator who, thanks to the use of suitable interfaces, gains the control of some actions of the SRLs. From the control point of view, it is fundamental to find the right trade-off between the degrees of freedom that are under the direct control of the user and the level of robot autonomy. The adoption of feedback interfaces can help the human to better command and use the SRL. In this review, we discuss all these aspects, relating them to the current literature on SRLs. We also present the main applications and the potential of these relatively recent devices, as well as the main neuroscientific questions they rise on the implications of their use on the users' body schema.
\end{abstract}

\section{Introduction}

Supernumerary robotic limbs (SRLs) are wearable robotic devices designed to achieve human sensorimotor augmentation. By sensorimotor or bodily augmentation we mean the enhancement of human physical capabilities, typically in manipulation and locomotion tasks. SRLs add artificial degrees of freedom to the human body, giving the possibility of performing more complex actions with increased strength and precision. In addition, SRLs enlarge the human reachable workspace. While SRLs aim at augmenting body functions, there are other technologies that target the cognitive augmentation of human capabilities leading to an 'improvement of the processes of acquiring/generating knowledge and understanding the world around us' [1]. Although the boundary between physical and cognitive augmentation might be blurred and the neurocognitive impact of the use of SRLs is still a subject of study [2,3], cognitive augmentation applications such as memory enhancement and complex problem solving [1] are out of the scope of this work.

While exoskeletons and prostheses can be considered augmentative technologies, too [4], SRLs have unique features and working principles. Differently from exoskeletons and exosuits, which are designed to mirror the kinematic structure of the body part on which they are worn and are used to empower human natural movements, SRLs represent additional degrees of freedom that need to be controlled independently from and/or simultaneously with biological limbs [5]. Although prostheses require control and feedback 
interfaces that share commonalities with those employed in conjunction with SRLs, they are different in nature with respect to SRLs, as they are developed to physically substitute missing limbs. Moreover, it is worth underlining that prostheses are not useful for patients with motor disabilities (e.g. paresis), whose limbs lost functionality, but are still present.

In less than the last decade, we have seen the development of SRLs with different usages (fingers, hands, arms, legs), actuation systems (fully actuated, underactuated), and design features (rigid/soft materials, level of anthropomorphism, etc). Besides the complexity of designing light and portable mechanical structures, it is important to consider the bilateral interface between the robot and the operator. Since the final aim of SRLs is to augment the manipulation or locomotion capabilities of humans, control signals from the human to the robot have to be acquired without interfering with the biological limbs by detecting, for example, human muscular activity, e.g. through electromyography (EMG) electrodes, or movements, e.g. through joysticks, accelerometers, magnetometers. Feedback from the robot to the human (visual, auditory, or haptic) is necessary to monitor the state of the SRLs.

Together with the design of novel devices, several applications of SRLs have been proposed, mostly falling into two main categories: augmentation of healthy individuals and compensation of motor abilities in impaired people. Both applications imply the sensorimotor augmentation of human skills. However, while when applied to people without motor impairments, SRLs allow the user to enhance his/her capabilities beyond the natural ones (e.g. carrying heavy loads or performing long overhead tasks), when applied to people with motor disabilities, SRLs compensate for missing functions of impaired limb(s), providing assistance when needed and substituting the impaired $\operatorname{limb}(\mathrm{s})$ from a functional point of view.

In this review, we give a 'device-driven' description of the field of SRLs. The mechatronic design (section 2), the control/feedback interfaces (section 3), and the applications (section 5) of SRLs are discussed depending on the considered type of robot, from supernumerary arms and legs to fingers and hands. A more 'problem-driven' classification of SRLs is available in a recent review [6], where, after a brief overview of the main devices, the discussion on design, control and applications is conducted by individuating general classes in which SRLs of different nature are included. Also the way we organized tables in our manuscript privileged a device-driven view. We believe that such a view can be beneficial to explain the evolution of SRLs over the years and to show that even if design and control problems for arms/legs and fingers/hands might be based on similar principles, they require different levels of complexity and have reached different stages in their development. Notice that sections 2, 3, and 5 of this review have the same structure. They start with an overview of the specific aspect and then the first two subsections are devoted to the literature review regarding arms/legs and fingers/hands, respectively. The final subsection summarizes the main trends derived from the literature analysis, giving some preliminary perspectives on the field.

In addition to robotics related considerations, SRLs pose several questions about the neurocognitive consequences of their use and, although not the primary focus of this review, we decided to give an overview of the main neuroscientific aspects related to the possible embodiment of SRLs (section 4). This aspect is not discussed in [6], while it is briefly reviewed in another recent work [7], which analyses SRLs developed until 2019 and classifies them based on the envisaged application and the adopted control method. Neuroscientific considerations on the 'Neural Resource Allocation' problem are the focus of [8], where only some works presenting methodologies for the assessment of sensorimotor control of SRLs are considered.

The field of supernumerary robotic limbs (SRLs) is relatively new and is still facing major implementation challenges. Perspectives on the field usually focus on possible ways of overcoming technical limitations in the design, sensing, and control of the devices [6, 7]. However, we think a broader view is needed, too. Future research on SRLs should not only focus on building SRLs, but also on developing sensorimotor interfaces to control them and receive feedback from them. The latter aspect is seldom treated in current literature, but we envisage that focusing on providing the right sensory stimuli to let users feel the SRLs as under their control could be the starting point for bringing human sensorimotor augmentation to the next level, in which humans can seamlessly control not only worn, but also grounded robots (see section 6).

The literature search at the basis of this work started in Scopus by searching in the Title, Abstract and Keywords the statement 'supernumerary AND robotic AND (limbs OR fingers OR arms OR hands OR legs)' and limiting the search to papers published by 2020. The publication year of the obtained results goes from 2012 to 2020 . We broadened the search by looking for 'supernumerary robotic limb' and then 'supernumerary robotic finger' (all of the words, anywhere in the article) in Google Scholar, year by year from 1980 to 2020. The results found for the years 1980-2011 were either unrelated to the topic or focused on neuroscientific aspects related to embodiment and body perception not directly applied to real robotic supernumerary limbs. An overview of the neuroscientific questions raised by SNLs is given in section 4, but a complete review of these aspects is out of the scope of this paper, which, instead, focuses on developed prototypes of SRLs, their control strategies, and their applications. 


\section{Design, actuation and sensing}

When designing SRLs, the choice of materials, actuators, and sensors usually aims at achieving a desired trade-off between wearability and dexterity of the robot. On the one hand, articulated and mostly rigid structures with as many degrees of freedom (DoFs) as degree of actuation (DoAs), i.e. fully actuated, can be very precise and reach a variety of configurations, but require a higher number of motors, sensors, and batteries, which can hinder the wearability of the device. On the other hand, light, compliant, underactuated structures can be more wearable at the price of reduced functionality and mobility.

On top of these considerations, safety is an essential principle to follow when designing SRLs, as they are meant to work in very close proximity to humans. This is why there is a growing trend towards the adoption of intrinsically safe soft structures, as discussed in section 2.3 .

This section describes how these challenges have been tackled so far in the development of supernumerary arms, legs, fingers and hands.

\subsection{Supernumerary arms and legs}

The development of supernumerary arms has paved the way towards robotic augmentation. These devices provide users with one or more additional robotic limbs which can move independently from the natural ones. Usually they are worn through a harness and designed to operate close to human limbs, possibly in areas that are unreachable by the user alone (e.g. overhead, behind the user, etc).

Several prototypes of supernumerary arms have been proposed in the literature. Most of them include a single extra limb (see tables 1 and 3), but there are also devices composed of two arms (see table 2). Two exemplary models are depicted in figure 1.

A first attempt to create a supernumerary limb is reported in [9], a patent presenting a modular wearable arm consisting of a sequence of articulating cubes. Each cube has a docking plate that enables the mechanical and electrical connection of modules.

In 2012, the research group led by Prof. Asada presented a pioneering work describing the design and biomechanical analysis of SRLs [10]. The concept was then studied in depth in subsequent works [11, 12], focusing on the control and the dynamic analysis of the SRLs. Obtained results led to the device presented in $[13,14]$, a novel type of wearable robot to assist workers in the assembling of an aircraft fuselage (figure 1(a)). It includes two supernumerary arms developed to hold objects, clamp them to a fixture, and guide and support human hands. An optimization method was introduced to identify the SRL kinematic configuration and joint torques that minimize the human workload.

Early work on supernumerary arms also focused on devices to be mounted on the user's shoulders $[15,16]$ and led to SRLs designed for overhead operations [17]. In [17], Bright and Asada developed an arm with a reduced number of DoFs (three) endowed with a soft Granular Jamming Gripper which can easily grasp objects from different directions without needing to be precisely oriented. The total weight of the device is around $5.5 \mathrm{~kg}$.

The ideas of helping users in accomplishing a manipulation task and supporting workers in tedious industrial scenarios were studied in [21] and [22]. Similarly to [14], these works aimed at helping users in tasks like hand-held objects and weight lifting.

Khodambashi et al [23] presented a fully actuated wearable arm with 4 DoFs designed and controlled to suppress residual vibrations of the SRL. The development of wearable robotic arms continued with Kojimal et al [24], who introduced an SRL based on a hybrid actuation system which is a combination of passive and active joints. Active joints embed an actuator thanks to which joint angles can be controlled automatically. Passive joints include a stopper mechanism and their joint angles can be changed manually when the joints are unlocked. This particular design aims at making the robot lighter, by reducing the number of actuators.

A novel concept of SRL to support the wearer when performing bi-manual tasks near the ground was proposed by Kurek et al [33] and extended in [37]. The robot has a control system for supporting the user with a desired impedance to effectively brace the upper body of its wearer when working near the ground.

In 2018, Saraiji et al [34] introduced the MetaArms, a couple of wearable anthropomorphic robotic hand-arm systems. The total weight of the device is about $9 \mathrm{~kg}$. Rather than focusing on the development of a novel arm, the research goal was to re-imagine what our bodies can do with the aid of wearable robotics using a body-remapping approach. Authors used the MetaLimbs [35], as a starting point for their hardware design.

With the aim of improving workers' ergonomics in industrial environments, Ciullo et al [36] proposed a wearable system composed of two passive gravity compensator arms and two soft robotic hands. The suite can be worn as a backpack, while the robotic hands are integrated thanks to a custom mechanical wrist interface. The arms can either support the user's upper-limbs, similarly to a passive exoskeleton, or work as 
Table 1. Table summarizing the main characteristics in terms of mechanical design, actuation system and control/feedback interfaces for single supernumerary robotic arms with mostly rigid structures.

\begin{tabular}{|c|c|c|}
\hline Device & Design & Control and feedback interfaces \\
\hline $\begin{array}{l}\text { Portable personal } \\
\text { wearable third arm } \\
(2007), \text { Eldershaw }\end{array}$ & $\begin{array}{l}\text { Fully actuated device with } 5 \text { DoAs. Structure } \\
\text { made of successive articulating cubes. Each cube } \\
\text { provides a single DoA and includes four infra-red }\end{array}$ & $\begin{array}{l}\text { Control. No specific control } \\
\text { algorithm. }\end{array}$ \\
\hline
\end{tabular}

et al [9]

Supernumerary

Wearable Robotic

Arm (2016), Khodam-

bashil et al [23]

Wearable Arm (2017),

Kojimal et al [24]

Shoulder-Mounted

SRL (2017), Bright and

Asada [17]

Brain-controlled

RLs (2018), Penaloza

et al [25]

Wearable Robotic Forearm (2018), Vatsal et al [27]

Orochi snake-like robot (2019), by Al-Sada et al [28]

Lightweight wearable arm (2019), Veronneau et al [30]

Lightweight Superlimb (2020), Guggenheim et al [22]

Reconfigurable SRL (2020), Zhang et al [32]

Two motors are used to actuate the roll and $z$ DoFs and a qb Robotics SoftHand Gripper is used at the end-effector.

The device is a reconfigurable series/parallel system using a cable-driven transmission with high accuracy, smooth transmission and low mass.
Two models: one has 3 actuated DoFs ( 1 at the elbow for horizontal panning, 1 for length extension, 1 for controlling a two-fingered gripper), and the other one has additional DoFs for vertical pitching of the arm and wrist rotation. Torque sensors embedded in the servo motors. Modular chain of servomotors with 25 DoFs. Different end-effectors can be attached to the arm.

Planar arm with 2 DoFs, attached on user's hip and powered by a MR-hydrostatic actuator, with low impedance. Newer version with 3 DoFs, MR clutches, hydrostatic transmissions and MRhydrostatic soft gripper [31].
Control. Methods for suppressing residual vibrations in the structures.

Control. By using the foot pose, or by tracking the big toe pose.

Control. Admittance control for the compensation of human movement.

Control. The arm is controlled through an EEG-based BMI. The robot is activated when the user imagines an action. Improved version of the control algorithm presented in [26].

Feedback. Auditive cues (bell sounds). Control. Two strategies: (1) voice commands, (2) Wizardof-Oz ('WoZ') setup where a remote operator directly controls the robot motion.

Control. Based on a small PC. It includes a movement generator and player system allowing users to create, save, and play back movements and sequences.

Feedback. Multiple haptic feedback strategies (e.g. taps-,gestures-,airfow-, brushing- and gripper-based) [29]. Control. For the 2 DoFs device, no specific control algorithm. For the 3 DoFs device, impedance control with virtual stiffness and damping coefficients tuned experimentally for each task to maximize dynamic response while giving a safe humanrobot interaction [31].

Control. Sensorized glove with force sensing resistors (FSR) mounted to each of the user's fingertips.

Control. No specific control algorithm.

supernumerary arms holding objects on which the user can operate with his/her limbs. In the first case, the robotic hands are commanded by the user to use and manipulate tools, in the second case the hands stabilize the grasped objects.

Vatsal et al in [27] developed a wearable robotic forearm for close-range human-robot collaboration. A major design consideration was the balance between the functional enhancements provided by the robot and the load on the wearer. In addition to the physical design aspect, an important area of exploration was the level of autonomy, and desired mode of interaction between the user and the robot. Two prototypes were developed, one with 3 and one with 5 actuated DoFs. 
Table 2. Table summarizing the main characteristics in terms of mechanical design, actuation system and control/feedback interfaces for devices including two supernumerary robotic arms.

\begin{tabular}{|c|c|c|}
\hline Device & Design & Control and feedback interfaces \\
\hline
\end{tabular}

SRLs (2012),

Davenport et al [10]

SRLs (2014),

Parietti et al [14]

MantisBot (2017), Kurek et al [33]

MetaArms (2018), Saraiji et al [34]
Two arms, each of which provided with 2 rotational joints at its base and 1 in the middle. Two fully actuated robotic limbs with 4 DoAs each, a waist bracket for securing the robot around the waist, and end-effectors attached to the tip of the arms.
Two limbs. Each limb has 2 active rotational DoFs at its shoulder and a passive linear DoF supported by an internal spring. Infra-red proximity sensors to measure limb lengths, 9DoF IMUs mounted on each SRL to measure roll and pitch.

Backpack including two robotic arms endowed with robotic hands, control boards, and batteries. An external PC is used for controlling the system. Each arm has 6 DoFs actuated by servo motors. A pressure sensor is attached to each thumb cushion and palm.

Commercial suite with two passive arms integrated with two soft robotic hands, with four passive dampers in between.

Device including two limbs with their endeffectors, and a backpack unit. Each limb has

5 DoFs.
Control. Low-level joint torque control.

Control. Algorithm that selects the optimal robot configuration given a limited number of available contact points with the environment and identifies the ground reaction forces that secure the drill jig to the aircraft structure, while minimizing the human workload. It also computes the joint torques necessary to create the desired static equilibrium.

Control. Control system designed and implemented on a proof-of-concept prototype for supporting the wearer with a desired impedance.

Control. Remapping of feet movements to move the end-effectors of the arms. The user's toes bending commands the closure of the robotic hands. Feedback. Force feedback to the foot sole provided by a motor-driven belt mechanism. Interaction method to alternate body scheme using artificial limbs substitution metamorphose presented in [35].

Control. Handle attached to the wrist interface used to proportionally control the opening/closure of the hands.

Control. No specific control algorithm. upernumerary for assembly (2019)

$\mathrm{Xu}$ et al [21] System (2018), Ciullo et al [36]

Table 3. Main characteristics in terms of mechanical design, actuation system and control/feedback interfaces for intrinsically soft supernumerary robotic arms.

\begin{tabular}{|c|c|c|}
\hline Device & Design & Control and feedback interfaces \\
\hline $\begin{array}{l}\text { Fabric-based robotic arm (2017), } \\
\text { Liang et al }[38]\end{array}$ & $\begin{array}{l}\text { Inflatable robotic arm made entirely } \\
\text { from TPU coated fabric. Miniature } \\
\text { diaphragm pump for actuation. } \\
\text { Miniature solenoid valves to control } \\
\text { the inflation. Air pressure sensors to } \\
\text { monitor inflating pressure. }\end{array}$ & $\begin{array}{l}\text { Control. Closed loop control scheme } \\
\text { with air pressure as feedback. }\end{array}$ \\
\hline $\begin{array}{l}\text { Soft Poly-Limbs (2019), Nguyen } \\
\text { et al [39] }\end{array}$ & $\begin{array}{l}\text { Fluid-driven, wearable, intrinsically } \\
\text { compliant arm made of elastomeric } \\
\text { materials. Newer version made of } \\
\text { fabric [18]. }\end{array}$ & $\begin{array}{l}\text { Control. Three user intent detection } \\
\text { modalities: (1) analog joystick, (2) } \\
\text { IMU worn on the back of a hand, and } \\
\text { (3) } 2 \text { EMG interfaces worn on the } \\
\text { biceps. }\end{array}$ \\
\hline
\end{tabular}

In 2019, Al-Sada et al [29] developed a modular wearable robot called 'Orochi'. It is a SRL designed based on three main considerations: multipurpose use, wearability, and unobtrusiveness in public. The robot consists in a chain of servomotors controlled by a small PC. Orochi can exploit multiple haptic feedback strategies (e.g. taps, gestures, airflow, and brushing) and the prototype is $240 \mathrm{~cm}$ long with 25 DoFs, a thickness of $42 \mathrm{~mm}$, and a weight of $1.4 \mathrm{~kg}$.

Veronneau et al [30,31] explored the design of wearable arms with the capability of performing dynamical tasks, differently from previous works, where only quasi-static motions were studied. The proposed SRLs can be attached on user's hip and are remotely powered by magnetorheological (MR)-hydrostatic actuators (see figure $7(\mathrm{c})$ ). The choice of such technology was based on the following criteria: (1) be lightweight, (2) be fast enough to compensate for human unpredictable motions, (3) be 


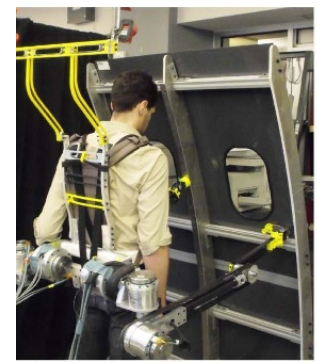

(a)

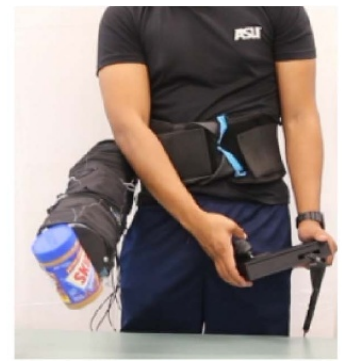

(b)
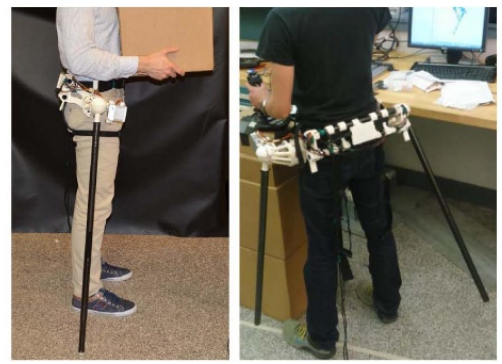

(c)

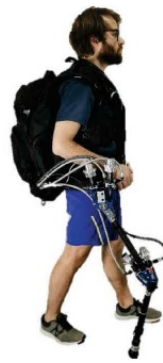

(d)

Figure 1. Examples of supernumerary robotic arms and legs. (a) Supernumerary arms for physical assistance; (c) [2014] IEEE. Reprinted, with permission, from [14]. (b) Supernumerary soft arm made of inflatable fabrics; (c) [2019] IEEE. Reprinted, with permission, from [18]. (c) Supernumerary legs for balance augmentation; ( [2017] IEEE. Reprinted, with permission, from [19]. (d) Supernumerary legs with portable power units. (c) [2020] IEEE. Reprinted, with permission, from [20].

strong enough to accomplish a multitude of tasks, (4) have high force-bandwidth and good back-drivability to control interaction forces between the user and the environment.

Devices presented so far have mostly rigid structures, sometimes endowed with passive compliant elements. Most of them have between 3 and 6 actuated DoFs [10, 14, 27, 35]. Those with few DoFs are typically used for holding or supporting parts [17], whereas the more complex ones can perform finer motions [35]. An alternative solution is to build completely soft SRLs (table 3), which can undergo continuous deformations. This approach was initially adopted in 2017 by Liang et al [38], who proposed a novel soft inflatable robotic arm entirely made of thermoplastic polyurethane (TPU) coated fabric. Preliminary results showed that the arm can achieve $150^{\circ}$ bidirectional bending, and the gripper can lift objects as heavy as $1.6 \mathrm{~kg}$. More recently, Nguyen et al $[18,39]$, presented the design and development of articulated, continuum, wearable, soft robotic arms called 'Soft Poly-Limbs'. The device presented in [39] is made of elastomeric materials, whereas the arm described in [18] is made of high-strength inflatable fabrics (figure 1(b)). Both robots are highly adaptable and can wrap around and hold objects of up to $3.8 \mathrm{~kg}$ and $11.13 \mathrm{~kg}$, respectively.

With the aim of assisting users in walking and balancing, Parietti et al [19, 40, 41] proposed a different approach to wearable robotics, developing a pair of robotic legs (figure 1(c)). Similarly, a couple of robotic legs made with lightweight carbon fiber tubes were developed by Khazoom et al [20]. A soft rubber end-effector was added to ensure a high friction coefficient to avoid slippage on the ground (figure 1(d)). The two power units, detailed in [31], weigh $3.5 \mathrm{~kg}$ each and can be worn in a backpack. The total mass of the robot is $9.7 \mathrm{~kg}$, which is lower than the $13.9 \mathrm{~kg}$ for of a human leg.

More complex devices are presented in [42], where two supernumerary fully actuated legs with 6 DoFs are developed. Differently from [20, 40], where the legs mostly work as balancing devices, the high number of actuated DoFs of this SRL allow it to have a greater configuration space and thus help the user in carrying heavy objects in an ergonomically correct way and in keeping different uncomfortable postures.

Further details on supernumerary robotic legs can be found in table 4 .

\subsection{Supernumerary fingers and hands}

In this section, we consider devices embedding a single finger, sometimes referred to as sixth fingers or third thumbs (see figure 2 and table 5), and devices including two or more fingers, up to the so-called third hands (see figure 3 and table 6).

The simplest, yet effective, way to augment the capabilities of a human hand is to add a single extra robotic finger to it. Whether it is worn on the palm or on the wrist, the finger can increase the grasping capabilities of the human hand and its dexterity even in complex actions (e.g. playing piano [58]).

Prattichizzo et al $[43,45]$ introduced the Robotic Sixth Finger, a modular, rigid, fully actuated device. The Robotic Sixth Finger is endowed with three or more DoFs for the flexion motion and one DoF for abduction/adduction. The device can be worn on the human wrist through an adjustable band and is provided with proprioceptive sensors (encoders in the joints). In [46], the finger has been enriched with force-sensing resistors placed on the finger's phalanges to allow for a more precise control by the human. Also a magnetic compatible version of the Robotic Sixth Finger device has been proposed by Hussain et al [44] with a main application in neuroscientific studies (see section 4).

Hussain et al [62] compared the fully actuated robotic sixth finger with an underactuated version of the device having only two motors, one for the flexion of the entire finger and one for abduction/adduction. The 
Table 4. Main characteristics in terms of mechanical design, actuation system and control/feedback interfaces for the supernumerary robotic legs and other limbs.

\begin{tabular}{|c|c|c|}
\hline Device & Design & Control and feedback interfaces \\
\hline Supernumerary robotic limbs for & Each of the two robotic legs has 3 & Control. Control strategies that enable \\
\hline balance augmentation (2015), & DoFs, two rotational and one linear. & the robot to stably assist the human \\
\hline Parietti et al [40] & $\begin{array}{l}\text { At the end of the robotic legs, end- } \\
\text { effectors with tunable spring-dampers } \\
\text { ensure safe and gradual interaction } \\
\text { with the environment. }\end{array}$ & $\begin{array}{l}\text { balance }[40,41] \text {. The control is based } \\
\text { on EMG signals from the torso and } \\
\text { two gaits have been identified for the } \\
\text { robot [19]. }\end{array}$ \\
\hline $\begin{array}{l}\text { Extra robotic legs (2018), Gonzalez } \\
\text { and Asada [42] }\end{array}$ & $\begin{array}{l}\text { Fully-articulated (6-DoF) robotic } \\
\text { legs worn at the back attached to the } \\
\text { person through a } 6-\mathrm{DoF} \text { force-torque } \\
\text { sensing interface. }\end{array}$ & $\begin{array}{l}\text { Control. Joint-level force and position } \\
\text { control, whole-body balance control. }\end{array}$ \\
\hline $\begin{array}{l}\text { Supernumerary leg powered by } \\
\text { magnetorheological actuators (2020), } \\
\text { Khazoom et al [20] }\end{array}$ & $\begin{array}{l}\text { Planar manipulator with } 2 \text { DoFs. The } \\
\text { leg is made with lightweight carbon } \\
\text { fiber tubes that minimize the mass } \\
\text { and inertia of the leg. The length of } \\
\text { the lower segment can be adjusted for } \\
\text { each user. A soft rubber end-effector } \\
\text { ensures a high friction coefficient to } \\
\text { avoid slippage on the ground. The } \\
\text { power units are detailed in [31]. }\end{array}$ & $\begin{array}{l}\text { Control. Impedance controller for gait } \\
\text { regulation. }\end{array}$ \\
\hline
\end{tabular}

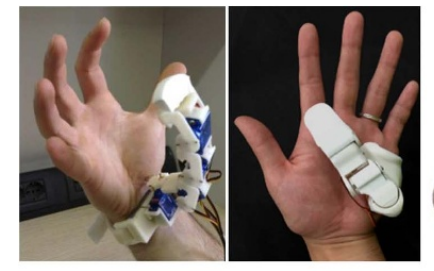

(a) (b)

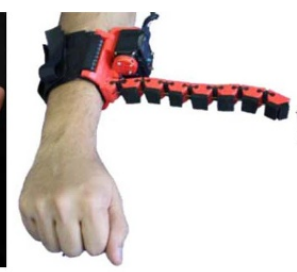

(c)

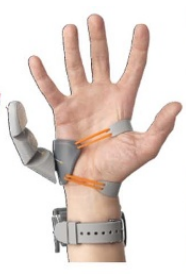

(d)

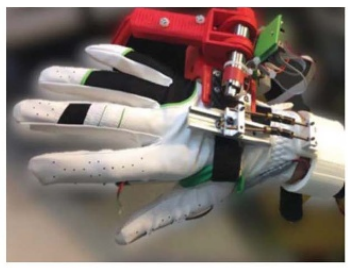

(e)

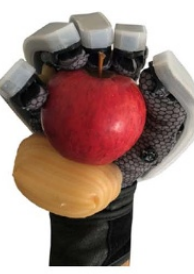

(f)

Figure 2. Prototypes of supernumerary robotic fingers. (a) (c [2014] IEEE. Reprinted, with permission, from [45]; (b) (C) [2015] IEEE. Reprinted, with permission, from [49]; (c) (C [2016] IEEE. Reprinted, with permission, from [53]; (d) Reproduced from [3]. CC BY 4.0; (e) () [2018] IEEE. Reprinted, with permission, from [57]; (f) Reproduced from [61].

motion is transmitted thanks to a nylon wire, which connects the phalanges with the motor placed at the base of the device. Springs are added between the phalanges to endow the finger with passive compliance and to bring it to its original position when required. While the underactuated finger results to be lighter, more portable and easily adaptable to different objects, the fully actuated version allows a more precise control of the finger trajectory and tighter grasps.

The solutions proposed above were mainly designed for hand augmentation, where the devices dexterity plays a major role. For assistive applications, the robustness to unwanted contacts with the environment and the ease of control are the most important goals [63]. For these reasons, Hussain et al introduced in [53] the Soft-SixthFinger, a soft-rigid version of the robotic supernumerary finger that has only one motor which actuates a variable number of joints through a tendon. The finger structure is made of modules composed of a rigid part acting as a link, and a flexible part acting as a joint. The compliance introduced in the system makes the device robust to impacts, light to wear, and easy to control, since it can passively adapt to objects with different shapes.

Recently, Hussain et al proposed a supernumerary robotic finger with an adaptive closed-chain structure inspired by the fin ray effect [59]. This design choice allows the finger to conform to the grasped objects and apply suitable forces to hold them stably.

The above described supernumerary fingers were developed by Prof. Prattichizzo's research group. Their main mechatronic features are listed in the second column of table 5 and extensively analyzed in [64], whereas some representative pictures are shown in figure 2.

The robotic thumb presented by Sobajima et al [49] is among the most relevant supernumerary fingers prototypes developed in recent years. It has been used in several studies as a platform for investigating different control and feedback modalities (see section 3). From the design point of view, the finger has three 
Table 5. Main characteristics in terms of mechanical design, actuation system and control/feedback interfaces for supernumerary robotic devices embedding one wearable finger.

Device Design, actuation and sensing Control and feedback interfaces

Robotic Sixth

Finger (2014),

Prattichizzo et al [43]

Extra Thumb (2015), Sobajima et al [49]

Soft-SixthFinger (2016), Hussain et al [53]

Morphology

Extension Kit (2018),

Leigh et al [56]

Third thumb (2018),

Cunningham et al [57]

Fin Ray sixth

finger (2019),

Hussain et al [59]

Pneumatic

supernumerary fin-

ger (2019), Singh et al

[60]

Telescopic finger (2020),

Gerez et al [61]

Third Thumb (2020),

Kieliba et al [3]
Fully actuated device with 4 DoAs (3 for flexion, 1 for abduction/adduction), modular structure made of ABS. Magnetic compatible version proposed in [44].

Fully actuated device with 3 joints (2 flexion, 1 abduction/adduction) and a force sensor at the fingertip. It is worn on the palm.

Underactuated device with 1 DoA for the flexion of 7 joints, tendon driven, modular structure with rigid links and flexible joints.

Interchangeable modules for building wearable robots. Rigid, modular structure covered with soft material.

Fully actuated device with 2 DoAs

(horizontal and vertical motions) and a rigid structure.

Linear actuator for the bending motion. Compliant structure with stiff crossbeams.

Soft finger with fiber-reinforced bidirectional pneumatic actuators.

Telescopic soft finger.

Tendon driven finger worn on the palm. 2 DoAs ( 1 for flexion and 1 for adduction/ abduction). Motors and batteries worn on the wrist and on the arm, respectively.
Control. Based on tracking data from the fingers of the augmented hand [43, 45], wearable buttons [46, 47], or two EMG interfaces worn on the arm [48].

Feedback. Vibrotactile ring [46, 47]. Control. Based on tracking data from the thumb of the opposite hand [49] or through EMG from auricularis muscles [50, 51].

Feedback. Tactile feedback through electrical stimulation to the opposite thumb [49, 50, 52], head mounted display to relay position information [50], vibrotactile feedback on the back of the hand wearing the device [51].

Control. EMG interface worn on the frontalis muscle $[53,54]$, or wearable buttons [55].

Feedback. Visual (LED lights) for EMG control [53], wearable haptic ring [55], vibrating feedback on the occipital area of the head for EMG control [54].

Control. The robot is controlled through software triggers.

Control. Horizontal movement controlled by the motion of the user's thumb of the augmented hand, vertical DoF controlled by the lifting of the left foot [57]. Both DoFs controlled by moving the foot [58].

Control. Push buttons.

Feedback. Visual (LED lights).

Control. Manual setting of the inflating pressure.

Control. Application installed in a smartphone.

Control. Pressure sensors taped underneath the big toes of the user's feet. The right toe controls the flexion, the left toe controls the abduction.

actuated DoFs and it is worn on the palm, opposite to the natural thumb. Two DoFs allow the flexion of the finger, whereas one allows abduction/adduction.

In 2018, Leigh et al [56] introduced the Morphology Extension Kit which includes three different types of modules (actuator, sensor, shape) that can be assembled to build a wearable finger. The resulting device can be worn in different positions around the human wrist or even temporarily detached from the user and externally mounted, according to the envisaged application. In the same year, Cunningham et al [57] presented a rigid wearable finger thought for augmenting the human hand to play music. The finger has 2 DoAs, one for vertical motion and the other one for horizontal motion.

More recently, innovative design techniques were introduced for building intrinsically compliant supernumerary fingers. Singh et al [60] developed bidirectional pneumatic actuators which can be assembled to form a soft robotic finger, whereas Gerez et al [61] presented a soft telescopic extra thumb embedded in an exoskeleton glove.

A collaboration between scientists and professional designers has led to the design of the Third Thumb [65], which has been used in neuroscientific studies on human augmentation [3]. It is a 3D printed, compliant, tendon driven device to be worn on the ulnar part of the palm. Motors and batteries are worn on other body parts. 


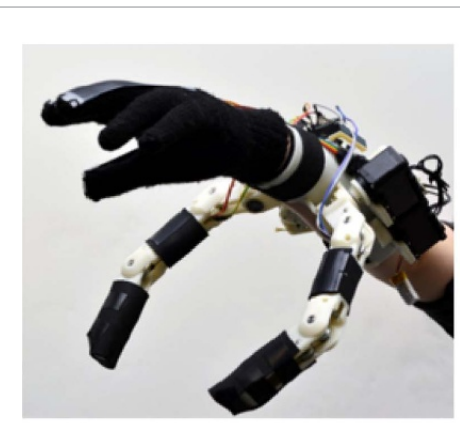

(a)

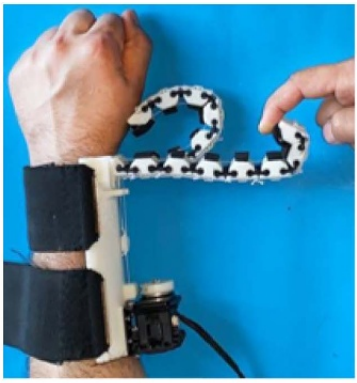

(b)

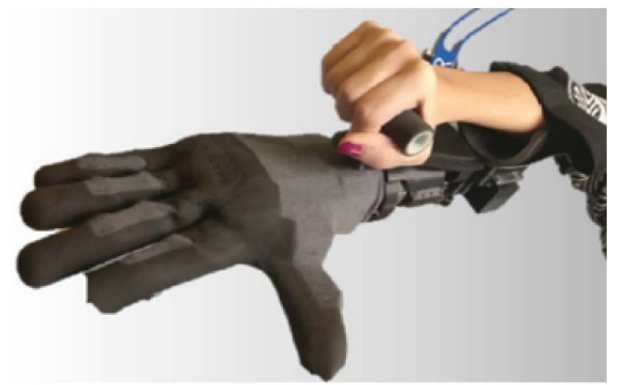

(c)

Figure 3. Supernumerary robotic devices with more than one finger. (a) Underactuated supernumerary fingers; (C) [2015] IEEE. Reprinted, with permission, from [71]. (b) Double Soft-SixthFinger; Reproduced from [75]. CC BY 4.0. (c) SoftHand X; () [2020] IEEE. Reprinted, with permission, from [80].

Table 6. Main characteristics in terms of mechanical design, actuation system and control/feedback interfaces for supernumerary robotic devices embedding two or more fingers.

\begin{tabular}{lll}
\hline Device & Design, actuation and sensing & Control and feedback interfaces \\
\hline $\begin{array}{l}\text { Supernumerary robotic } \\
\text { (SR) fingers (2014), Wu }\end{array}$ & $\begin{array}{l}\text { Fully actuated, two fingers having 3 DoAs } \\
\text { each. A newer version embeds force sensors } \\
\text { and Asada [67] }\end{array}$ & $\begin{array}{l}\text { Control. Based on tracking data from the } \\
\text { fingers of the augmented hand [67, 69] or } \\
\text { an IMU [68]. }\end{array}$ \\
$\begin{array}{l}\text { FR fingers with soft } \\
\text { Fully actuated, two fingers having 2 DoAs }\end{array}$ & $\begin{array}{l}\text { Control. Based on tracking data from the } \\
\text { inclusions (2015), Ort }\end{array}$ & $\begin{array}{l}\text { each. Additional DoA at the base for trans- } \\
\text { fingers of the opposite hand. }\end{array}$
\end{tabular}
et al [70] lation. Textured rubber pads and small air bubbles to increase friction and compliance. Underactuated device, two fingers hav-

Underactuated SR fingers (2015), Wu and Asada [71]

Pneumatic SR fingers (2017), Tiziani et al [72]

Double Soft-SixthFinger (2017), Hussain et al [74]

Otariidae-inspired supernumerary flippers (2020), Liu et al [76]. ing 2 DoAs and 3 DoFs each, cable driven, embedded force sensors.

Three fingers with soft rigidizable phalanges and variable stiffness pneumatic bending actuators. Newer version of the device with two fingers presented in [73].

Underactuated device, two fingers, tendon driven, modular structure with rigid links and flexible joints. Two DoAs, one per finger [74], or one DoA with a differential mechanism [75].

Two fingers, 8 DoAs each, soft pneumatic actuators reinforced by fabric inspired to origami and kirigami. Pressure sensor for each actuator.
Control. Method exploiting both the grasp synergy and the redundancies in the elbow.

Control. Manual setting of internal pressure.

Control. EMG interface worn on the frontalis muscle [74]. Feedback. Visual (LED lights) for EMG control [74].

Control. Cascaded control structure: posture-control outer loop, several pressure-control inner loops [77].

Pictures of some of the above described fingers are shown in figure 2, whereas their main design features are listed in the second column of table 5 .

It is worth mentioning that other works not primarily focused on augmentation actually developed structures that can be exploited as supernumerary fingers. Agharese et al [66], for example, presented HapWRAP, a pneumatic wearable robot which can grow around the human wrist and provide haptic feedback.

Supernumerary limbs with two or more robotic fingers allow users to perform stronger and more stable grasps. However, bulkier and heavier structures can hinder the functioning of these devices and a trade-off between strength and wearability needs to be achieved.

A fully actuated device with two wearable supernumerary robotic (SR) fingers was presented by Wu and Asada in 2014 [67]. The two SR fingers are fixed to opposite sides of the wrist and each of them has 3 DoFs. One finger can perform circumduction, abduction, and flexion, similarly to a thumb, and the other one has one DoF for abduction and two for flexion. A recent version of the device embeds force sensors in the fingertips and an inertial measurement unit (IMU) on the wrist [68].

In 2015, Ort et al introduced a wearable device with two SR fingers with soft inclusions [70]. Each finger is fully actuated with 2 DoFs for flexion. The device includes an additional DoF in the base of the fingers to enable translation along the bottom of the forearm. This allows the user to adjust the workspace of the SR 
fingers to the task at hand. Textured rubber pads and small air bubbles on the fingers were added to increase friction and compliance.

An underactuated prototype of SR fingers was developed by Wu and Asada to simplify the control and make the structure lighter [71] (figure 3(a)). It consists of two fingers having 2 DoAs and 3 DoFs each (2 for flexion/extension, 1 for abduction/adduction), with an actuation systems based on cables. The fingers also embed force sensors to monitor contact forces with the grasped objects.

In 2017, underactuated and compliant devices with multiple fingers were developed by Hussain et al [74] and Tiziani et al [72]. In [74], two Soft-SixthFingers [53] were combined into a single device to allow the lifting of heavy objects and improve the stability of the achieved grasps. Recently, Malvezzi et al proposed a differential mechanism to actuate the double Soft-SixthFinger with only one motor [75] (figure 3(b)). In $[72,73]$, fingers with soft rigidizable phalanges and variable stiffness pneumatic bending actuators manufactured using soft lithography fabrication methods were presented.

Very recently, a soft device embedding two hand appendages inspired by flippers of otariids has been presented [76]. Thanks to a structure composed of multiple soft actuators, the fingers have increased dexterity and load-to-weight ratio.

Several works have addressed the challenge of adding more than one robotic finger to the human hand, but only a few articles have explicitly focused on creating a third, anthropomorphic hand. In 2018, Ciullo et al [78] integrated a soft robotic hand with a passive arm support and conducted a preliminary study to find the optimal position of the extra hand with respect to the human one. The supernumerary hand system is called SoftHand X and derives from the SoftHand [79], a tendon driven underactuated anthropomorphic hand with 1 DoA and 19 DoFs. In [80], the hand-arm system design (figure 3(c)) was upgraded and used to compensate grasping functions in stroke patients (see section 5). While supernumerary fingers typically form a 'hybrid human-robot grasp' with the user's limb, the SoftHandX is designed to pick up objects autonomously, similarly to a prosthesis. However, like SRLs, the supernumerary hand by Ciullo et al adds DoFs to the human body and substitutes an impaired limb from a functional point of view, without a complete physical substitution. In addition, the paretic hand of the patient still plays a role in the task, as it supports, and possibly activates, the robotic device through residual motions. These features make the SoftHandX closer to a SRL than to a prosthesis, and open to the possibility of applying it to enhance the capabilities of healthy people, possibly in conjunction with suitable supports [36].

The main design features of the above described supernumerary limbs embedding two or more fingers are summarized in the second column of table 6 and pictures of some exemplary prototypes are shown in figure 3.

\subsection{Concluding remarks}

The quest for wearability and lightness has driven the design of most of the SRLs presented in the literature, and is especially important for supernumerary arms and legs, for which achieving a trade-off between weight and dexterity is still an open challenge. The adoption of remote actuation systems and the development of intrinsically soft structures are proposed as possible promising future directions, but they require rather structured environments. It is expected that future design solutions will be highly application-based and with a complexity proportional to the needed dexterity.

Prototypes of supernumerary fingers, instead, already have acceptable levels of wearability. Their mechatronic design has evolved according to the envisaged applications and based on technological and methodological advancements in the field of robotics. Similarly to what happened for exoskeletons [81], research on soft robotics, for example, has boosted the development of some of the latest models of SRLs, leading to intrinsically soft devices. It is expected that soft and robust solutions with a low number of actuators will be preferred for devices designed for assistive applications (section 5), whereas more dexterous and complex structures will be designed to augment human manipulation abilities. Other emerging trends in the design of supernumerary fingers include modularity and reconfigurability which allow the easy adaptation of devices to different applications and users [82].

\section{Control and feedback interfaces}

The selection of control interfaces and strategies is closely related to the adopted SRL design. The more actuators and sensors the device is endowed with, the more accurately it can be moved. The presence of several actuated DoFs implies a larger configuration space for the robot and usually requires the implementation of high level control strategies that allow users to control the coordinated motion of the DoFs of the SRLs, and not the single DoFs [43]. A trade-off should be found between what the user can actually command and the level of autonomy of the robot. The availability of proprioceptive and exteroceptive sensors, for example, allows the implementation of algorithms for the recognition, the 
prediction, and possibly the autonomous compensation of human motions for enhanced collaboration and safety [17].

SRLs with a low number of actuated DoFs are usually easier to control from the user point of view, exploiting, for example, wearable switches [47]. In simpler devices, underactuation and limited sensing capabilities are typically compensated by an increased compliance of the structure, which can easily adapt to the surfaces touched by the SRL, and does not require additional safety features.

As SRLs are designed to work in coordination with natural limbs, a major challenge in their development is to devise control interfaces and strategies that do not interfere with the activity of human limbs. Control interfaces should be unobtrusive, while control strategies should allow a seamless collaboration between robotic and biological limbs.

SRLs are wearable devices and as such they automatically provide a haptic feedback on the user body which directly feels not only the presence (weight) of the robot, but also part of the interaction forces that arise between it and the environment $[17,83]$. In this respect, important research questions include whether or not to provide the human with additional feedback cues, and which type of cues to adopt. Using a feedback interface adds complexity and weight to the whole system, but can be very beneficial for enhancing the user perception of the SRL state.

This section describes the main methods employed in literature for interfacing SRLs with their users. While there are also works that focus more on regulating the autonomous behavior of the SRL implementing a so-called 'indirect-control' [7] (e.g. to compensate for human motions [17] or to support the human body $[20,41])$, the main focus here will be on papers presenting devices that allow the user to command the SRL. In this case, two main control and feedback modalities can be identified, as explained in table 7.

\subsection{Supernumerary arms and legs}

How to easily, intuitively, and effectively control supernumerary limbs is a challenging research question, above all when dealing with wearable arms and legs, which are complex and voluminous devices. The control interface for such mechanisms should be wearable, portable, or easy to be integrated in a structured environment. To this end, multiple solutions involving different body parts have been developed. The main interfaces presented in the literature for supernumerary arms and legs are briefly summarized in tables 1-4 and some representative examples are shown in figure 4.

In $[24,34,84]$ the foot is selected as a preferred location for the control interface (see an example in figure 4(a)). The common idea is to track the user's foot position/rotation and/or toe posture and move supernumerary robotic arms accordingly. An IMU-based control algorithm was exploited also in [39] for tracking the hand and using its orientation as control input.

More complex systems involving electroencephalography (EEG) based control were pioneered by Penaloza et al $[25,26]$. The authors presented a human-like robotic limb that can be activated through an EEG-based brain-machine interface (BMI) when the human operator imagines a grasping action. The system has visual context awareness capability allowing it to recognize objects and human actions. The user study detailed in [25] serves as a proof of concept and opens up the possibilities to explore more complex experimental scenarios.

Also Nguyen et al [39] used physiological signals, but this time acquired through sEMG sensors, for controlling a soft robotic arm.

Vatsal and Hoffman [27] performed a pilot human-robot interaction study comparing direct voice-control with autonomous control (Wizard-of-Oz setup), where a remote operator directly controlled the robot motion, unbeknown to the users [85]. The study revealed that autonomy is more task-time efficient and preferred by users when compared to voice-control.

Guggenheim et al [22] exploited human DoFs redundancy for communication and control. Indeed, fingers are often redundant for performing a task, e.g. holding a box. Although both hands are busy, some combination of the finger forces is still available for generating signal patterns. The authors developed an algorithm and a sensing glove (figure 4(c)) for generating coded finger force patterns without interfering with the performance of the primary task.

A more intuitive control technique is presented in [28]. Al-Sada et al developed a graphical user interface that provides easy access to SRL control parameters. It also includes a movement generator and a player system that allows users to create, save, and play back movements and sequences.

Some works rely on more standard and widespread control systems, like analog joysticks, as described, for example, by Nguyen et al [39].

For what concerns the control of supernumerary legs, while in [41] the focus was on regulating the autonomous behavior of the robot to stabilize it while supporting the user's body, in [19], authors aimed at creating an interface as simple and intuitive as possible to let the human control SRLs. In particular, an association between torso EMG signals and robot DoFs was identified: the activation of the muscles on the 


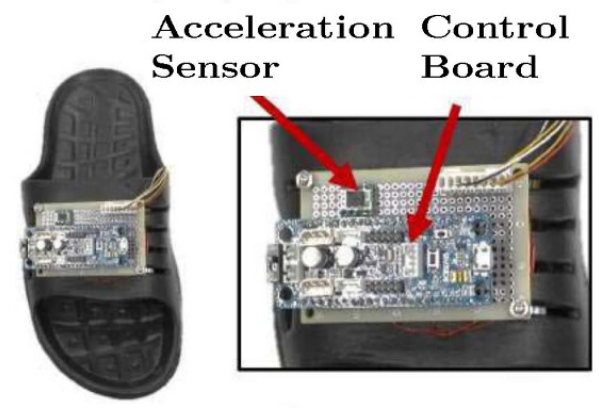

(a)

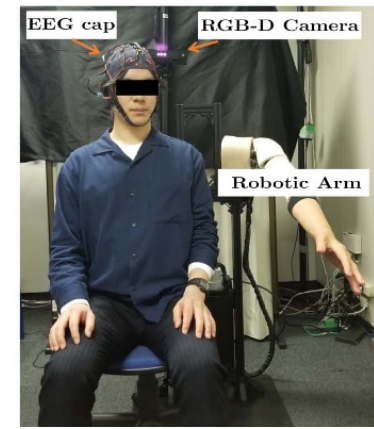

(b)

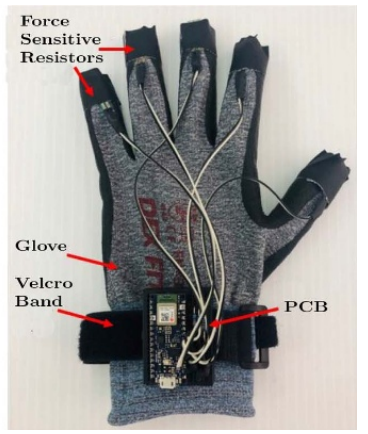

(c)

Figure 4. Control interfaces for supernumerary robotic arms. (a) User interface exploiting the foot pose; (c) [2017] IEEE. Reprinted, with permission, from [24]. (b) User wearing an EEG cap for controlling a supernumerary arm while an RGBD camera acquires environmental visual information; (C) [2018] IEEE. Reprinted, with permission, from [25]. (c) Glove with force sensing resistors (FSRs) mounted in each fingertip; (c) [2020] IEEE. Reprinted, with permission, from [22].

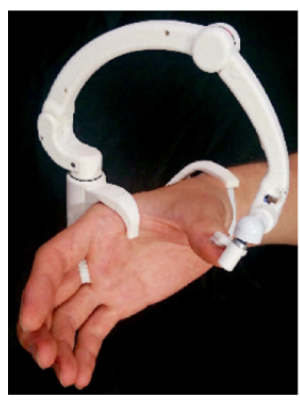

(a)

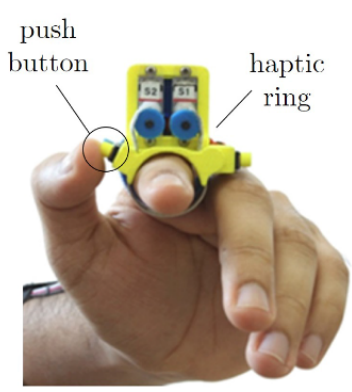

(b)

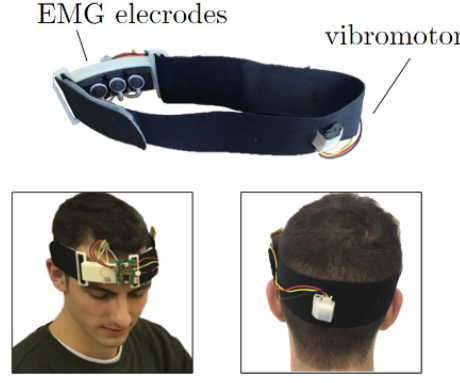

(c)

Figure 5. Wearable interfaces for supernumerary robotic fingers. (a) Thumb motion capture device with four encorders: a motion of the right thumb is mapped onto the motion of a supernumerary robotic finger worn on the left hand. () [2015] IEEE. Reprinted, with permission, from [49]. (b) Push buttons embedded in a ring worn on the opposite hand. (C) [2017] IEEE. Reprinted, with permission, from [55]. (c) EMG headband detecting the activation of the frontalis muscle. (C) [2019] IEEE. Reprinted, with permission, from [54]. The three interfaces also provide tactile feedback cues: (a) electrical stimulation, (b) vibration and normal forces, (c) vibration.

right side of the user controlled the right robotic limb, while the muscles on the left side controlled the left robotic limb. Three control strategies (position control, velocity control, force control) have been implemented to determine how EMG signals translate into joint torques. The same EMG-based control scheme was adopted in [86] to study the coordination between natural and artificial limbs.

Differently, in [20], a controller is designed to apply a varying force on the ground and swing back into position for the next step cycle. This is achieved using a reference generator that feeds the end-effector reference position to an impedance controller and that generates open-loop torque commands to push on the ground in synchrony with the walking gait cycle.

Only a few works investigated the use of feedback interfaces for supernumerary arms. Penaloza et al [26] exploited auditive cues to inform the user about the actual status of the robot (figure 4(b)). Al-Sada et al [29], developed the HapticSnakes, which is a waist-worn snake-like multi-robot system that can deliver a variety of haptic stimuli (vibrotactile, tangential and shear forces), and varied feedback in VR (e.g. fans to generate airflow). Lastly, in [34], force feedback to the foot is provided by a motor-driven belt mechanism. Two motors are placed in the interface and used to move a belt wrapped around the user's foot. In this way, the user can feel force feedback according to the pressure value reported by the associated robotic hand touch sensors.

\subsection{Supernumerary fingers and hands}

An overview of the devices analyzed in this subsection is provided in the third column of tables 5 and 6 , whereas some exemplary prototypes are shown in figure 5.

Wearable supernumerary fingers and hands require control and feedback interfaces that are wearable as well, or at least portable. Most of the interfaces presented in literature are designed to be worn on upper limbs (e.g. hands, arms), or other body parts (e.g. foot, forehead). Only one of the analyzed papers goes in the direction of portability, proposing to control an extra finger through a smartphone application [61]. 
Early works on supernumerary robotic fingers focused on coordinating the closure of the robotic fingers with that of the biological ones, applying a synergistic approach. In $[43,67,71]$, a dataglove is used to track the motion of the human hand and coordinate the closure of the supernumerary fingers (figure 3(a)).

Other papers introduced interfaces to be worn on the opposite hand embedding either tracking devices to monitor gestures (e.g. thumb interface [49] (figure 5(a)), dataglove [70]), or push buttons to trigger motion primitives in the robotic finger (e.g. ring interfaces $[46,55,59]$, figure 5(b)).

Wu and Asada proposed to implicitly control and adapt the actions of two supernumerary fingers by monitoring the movement of the human arm with IMUs. In [71], a redundant motion of the elbow was used to perform 'hold-and-manipulate' tasks, whereas in [68] a method to compensate for disturbances induced by human motions was presented.

The Third Thumb introduced by Cunningham et al [57], is controlled by two human motions, either coming from different body parts (thumb of the augmented hand and foot [57]), or coming both from the same limb (foot [58]). Pilot tests conducted with the third thumb showed that controlling the device demands high coordination and cognitive load at the beginning, but these aspects can be mitigated thanks to training [57]. In addition, it was shown that task performance mainly depends on the ability of the user to move the limb wearing the interface [58].

A foot interface is used also in [3], where the flexion/extension and adduction/abduction of a supernumerary finger are commanded by exerting a pressure with the right or the left toe, respectively. The motion of the finger is proportional to the applied pressure. While the control interface presented in [58] is based on an IMU sensor worn on a single foot, the interfaces used in [3] employ pressure sensors worn underneath two different feet.

Besides human kinematic signals coming from simple or more complex body motions, also muscular activity can be used to command supernumerary fingers. To this aim, interfaces based on surface electromyography (sEMG) have been developed. sEMG is a non-invasive method to detect electrical signals generated during a muscle contraction. It provides information at different levels of detail, from global muscle activity and fatigue, to single motor unit recruitment. sEMG has been used in several applications, from sport to rehabilitation and prostheses control, and has been often implemented in wearable devices. When it comes to supernumerary fingers, EMG based interfaces are particularly suitable as they can be placed in different body parts and usually allow a rather intuitive control.

Hussain et al [48] used two EMG interfaces for controlling a fully actuated supernumerary finger. One was used for commanding the robot motion (Myo Armband, ThalmicLab), and the other one (customized EMG interface with one channel) was employed for tuning its compliance.

While fully actuated fingers may require complex interfacing systems to exploit all their features [48], when using soft underactuated fingers like the Soft-SixthFinger, it is more convenient to have simpler interfaces that trigger the opening and closing of the device. In $[53,54]$, for example, the Soft-SixthFinger is controlled through a single EMG interface worn on the forehead. The EMG electrodes capture patterns of contractions of the frontalis muscle (activated with an upward movement of the eyebrows) to open and close the finger (figure 5(c)). This solution does not hinder the use of the opposite hand and is particularly suitable for assistive applications (see section 5).

Meraz et al [50] and Shikida et al [51] proposed the use of sEMG signals from posterior auricularis muscles to control an extra thumb. In particular, signals coming from the muscles behind both ears are used to control either the planar position of the fingertip [50] or the rotation of the base joint of the finger [51]. Posterior auricularis muscles are sometimes not easy to activate, but are suitable for the control of an extra limb because they are vestigial muscles without a predefined functionality.

Regarding the control of supernumerary hands, Ciullo et al introduced different interfaces, from a lever that proportionally closes the hand [78], to input devices detecting applied force, finger bending or trigger activation [80].

Alongside with input interfaces, also feedback interfaces have been proposed in the literature to enhance human control of supernumerary robotic fingers.

Hussain et al [46] proposed a vibrotactile ring to inform the user about task related events (e.g. making/braking contact, reaching of actuators limits) or quantities (e.g. intensity of the force exerted by the finger). The adopted supernumerary finger was endowed with force sensors on the phalanges to acquire the needed information. An enhanced version of the haptic ring was presented in [55], where patterns of vibrotactile cues were used to alert the user about relevant events, while skin indentation was employed to transmit the force exerted on the object (figure 5(b)). In both papers, experiments showed that haptic feedback is appreciated by the users and significantly improves task performance in terms of exerted force on the object, and perceived effectiveness. 
Table 7. Motor control and sensory feedback for SRLs: main terms and concepts.

\begin{tabular}{|c|c|c|}
\hline \multirow[t]{2}{*}{ Motor control } & Continuous type & $\begin{array}{l}\text { The control of the SRL continuously depends on the measurements of the } \\
\text { human body. }\end{array}$ \\
\hline & Event-driven type & $\begin{array}{l}\text { The SRL moves along predefined primitives and is controlled by events } \\
\text { triggered by the human body poses or gestures. }\end{array}$ \\
\hline \multirow[t]{2}{*}{ Sensory feedback } & Continuous type & $\begin{array}{l}\text { The haptic feedback for augmentation is continuously displayed to the human } \\
\text { body during the entire task execution. }\end{array}$ \\
\hline & Event-driven type & $\begin{array}{l}\text { The haptic feedback for augmentation is chosen among a finite set of } \\
\text { predefined primitives in correspondence of events related to task execution. }\end{array}$ \\
\hline
\end{tabular}

In $[53,54]$, the human is informed about the correct EMG command detection through visual and vibrotactile feedback, respectively. This acknowledgement can reduce the muscular effort and time needed to perform a sequence of actions with the supernumerary finger [54].

Different feedback interfaces have been proposed for the Extra Robotic Thumb developed by Sobajima et al [49]. In [49, 52], tactile feedback through an electrical stimulation device worn on the opposite thumb was used to convey information about the force exerted at the tip of the robotic finger. In [50], a head mounted display was employed to relay information about the position of the thumb, whereas in [51] authors proposed to stimulate the back of the augmented hand with vibrotactile feedback according to the change of the base joint angle.

\subsection{Concluding remarks}

Having suitable control and feedback interfaces is fundamental to achieve a smooth integration between humans and SRLs. Several devices have been presented in the literature. Most of them are wearable and designed to fit different body parts, e.g. rings, armbands, headbands, while others are portable, e.g. based on joysticks or on mobile applications.

In most of the cases, human motions and muscular activity are exploited to keep motor control over the supernumerary limb(s), whereas the haptic channel is exploited for sensory feedback. While the use of feedback interfaces is rather common in supernumerary fingers, it is still rare in supernumerary arms and legs.

When the user can command the robot through an ad-hoc interface, control and feedback strategies employed in the literature can be classified according to table 7 , where we refer to haptic feedback as it is the most used one.

In the continuous motor control, the reference trajectory for the robotic limb depends on continuously tracked motions of the human body (e.g. synergistic control of supernumerary fingers [43, 67], remapping of user's foot-and-toe movements on the motion of supernumerary arms end-effectors [34]). In the event-driven mode, the occurrence of an event drives the evolution of a finite state machine selecting a motion primitive within a finite set, which in turn defines the reference trajectory for the robot. The event can be triggered by either a physical switch on the interface [55] or by the detection of a posture, gesture or contraction of the human body [39, 54, 70].

We distinguish between two types of feedback, depending on their nature: event-driven and continuous. The first type is particularly suitable for improving operator's awareness during task execution, through the notification of relevant events, including constraint and singularity proximity, task completion, and robot understanding of human commands or intentions [54, 87]. Continuous feedback proves most appropriate in tasks for which a high dexterity degree is required, because it continuously informs the user during the entire task execution. In [55], for example, continuous haptic feedback is used to display the force applied on the grasped object. This information can help the user to decide when to lift the object, preventing possible slippage.

Future research efforts will focus on increasing the intuitiveness and unobtrusiveness of control interfaces for SRLs, with the main aim of reducing the cognitive burden requested to users. As detailed in section 6, we think haptic feedback can play a key role in enhancing the users' ability of commanding SRLs in conjunction with their natural limbs.

\section{Neuroscientific considerations}

In individuals born with six-fingered hands, a rare genetic condition known as polydactyly, the control of the additional finger relies on an innate neural hardware that includes additional dedicated muscles and nerves, as well as dedicated representations at cortical level, either in the motor and sensory cortices [88]. Such hardware allows polydactyly individuals to easily control over-physiological degrees of freedom, to an extent that they can even improve some manipulation abilities without particular efforts [88]. 
The control and the use of 'acquired supernumerary limbs' open an unprecedented field of research in neuroscience, as well as conceptually new rehabilitative scenarios. Literature in this sense is still relatively limited, but it is not difficult to predict a forthcoming increase of research in the next years.

There are at least two main scientific questions that are starting to be addressed: (1) will humans use the same or alternative motor synergies for using supernumerary limbs? (2) Will these devices, that widen the action space of the individual (i.e. the peripersonal space), impact on the extant body schema representation?

The two questions fall into the broad concept of 'brain plasticity', that is the ability of the brain to continuously adapt to environment changes. Motor synergies represent 'the output patterns of conjoined muscle activity whose timing and amplitude modulation enable the correct production of goal-directed movements' $[89,90]$. In this way, the motor system organizes brain-muscles commands in a simplified way by a computational perspective. In order to finalize a grasping or a pinch-grip action, some hand/forearm muscles groups should be activated and others should be inhibited, simultaneously. A recent study [91] has been the first to verify these patterns of muscular activations/deactivations during the use of a supernumerary robotic thumb in the context of a motor imagery task. Authors measured the corticospinal output by observing amplitude changes of motor evoked potentials from intrinsic and extrinsic hand muscles elicited by transcranial magnetic stimulation (TMS) of the primary motor cortex (M1). Indeed, TMS is a unique primer [92] allowing the direct assessment of corticospinal function in imagined or executed actions [93]. After a few minutes of training with the robotic finger, new bioartificial corticospinal synergies emerged, supporting the different motor strategies for pinching and grasping triggered by the presence of the additional thumb. These changes, that represent a system-level form of brain plasticity, demonstrate that the brain is open to adopt very quickly new interactions with objects located within the peripersonal space.

Such an 'augmentation plasticity' [94] may represent the substrate by which humans would prefer to use an additional hand to accomplish demanding manipulative tasks [95] and it also opens new questions, that can be summarized in the following conceptual framework. As the brain works in an integrated manner, it is unlikely that adapting changes will involve only the motor system, leaving unaltered the sensory representation we have of our body. The so called 'body schema' is a high-level, continuously updated hypothetical construct, through which we register our posture (or body part position) in relation to the peripersonal space [96], that in turn is obviously modified by the supernumerary finger (or limb). The body schema is highly adaptable. The level of dexterity in tools use, for example, improves once the tool has been incorporated, or embodied, into it [97]. A supernumerary finger is conceptually similar to a common tool, and there is evidence that it can actually be embodied into the user's body schema [98], even when presented as an avatar in a virtual reality scenario [99]. Moreover, the use of haptic feedback seems to promote the embodiment process [100].

Preliminary data based on functional magnetic resonance imaging (fMRI) during the use of a magneto-compatible version of the Robotic Sixth Finger (see section 2) indicated that healthy subjects showed increased activations in brain regions relevant for motor control, but even for body space [44]. In another study, Kieliba et al [3] found that subjects trained for five days to use a 'third thumb' improved some motor task abilities involving the six fingers of the trained hand. These abilities persisted even in absence of visual feedback and remained stable by increasing subjects' cognitive load. Trained subjects reached higher scores when interrogated to four statements relating to key embodiment features (body ownership, sense of agency, body image, somatosensation). Interestingly, subjects scanned with fMRI after the five-day training showed a shrinking of the representation of the augmented hand in the contralateral M1 (i.e. Broadman area 4), possibly reflecting the fact that the particular 2-DoF third thumb utilized in this study resulted in breakage of the natural finger coordination patterns (kinematic synergies) of individual fingers. As a consequence, this fact might have contributed to 'disorganize' the cortical representation of the natural hand within M1. This could theoretically represent a form of maladaptive plasticity [2], that however was transient, as it disappeared in subjects re-scanned about ten days later, and more importantly without behavioral consequences, as it did not interfere with the use of the augmented hand [3].

\section{Applications}

Thanks to robotic augmentation, humans are expected to perform a greater number of tasks than what they can do with biological limbs only. In this section, we present a set of paradigmatic examples sufficient to analyze the most representative and relevant applications that we envisage in the near future for human augmentation by SRLs. As depicted in figure 6, SRLs may have applications for both disabled and healthy people. On the one hand, patients with motor impairments can improve their quality of life by using SRLs to compensate for most of the lost motor functions (blue arrows, figure 6). On the other hand, SRLs may open new horizons in collaborative robotics by giving to the human enhanced abilities to perform complex tasks, having gained the control of the robotic extra limbs (red arrow, figure 6). 


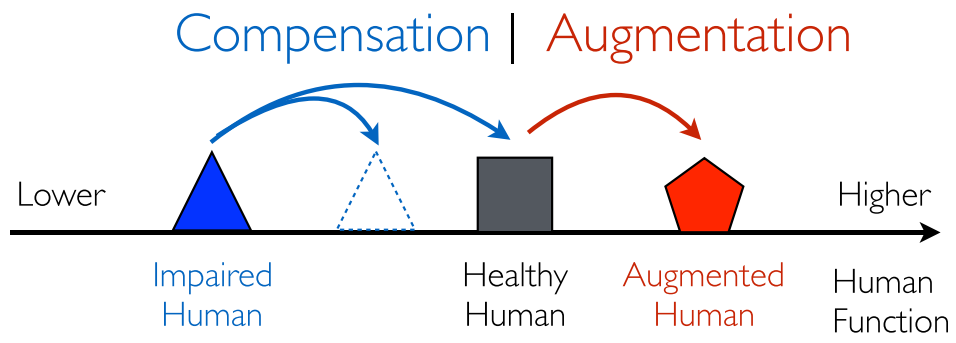

Figure 6. SRLs can find applications for both disabled and healthy people. For the first, augmentation corresponds to the compensation (blue arrows) of lost motor abilities, that can be partially (dotted triangle), or totally, recovered. For the second, augmentation (red arrows) leads to get skills that go beyond the biological ones (augmented human).

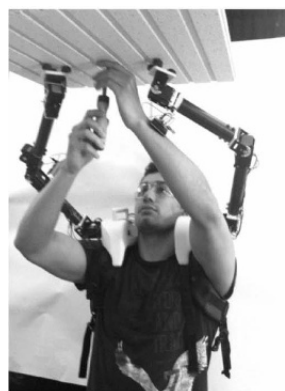

(a)

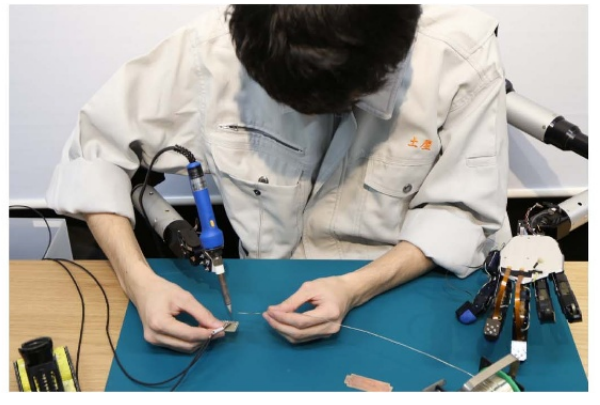

(b)

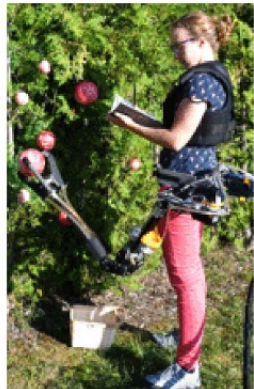

(c)

Figure 7. Applications of supernumerary robotic arms: ((a) overhead assembly, @ [2021] IEEE. Reprinted, with permission, from [83]; (b) welding [35]; (c) fruit picking, (c) [2020] IEEE. Reprinted, with permission, [31]).

Examples of applications for supernumerary arms and fingers are shown in figure 7 and figure 8, respectively.

\subsection{Applications for arms and legs}

A promising application of supernumerary robotic arms is the manufacturing of large objects (e.g. assembling planes $[14,17])$. This activity requires human workers to perform extremely complicated and fatiguing tasks (e.g. assembling electromechanical systems, drilling holes, inspecting structures) in uncomfortable positions (e.g. overhead tasks $[83,101]$, figure $7(\mathrm{a})$ ), and the strength and versatility of many of the proposed supernumerary arms (see section 2) can be particularly useful in such situations.

Additionally, workers might also be required to operate on elevated platforms or on scaffolds, which expose them to the risk of slipping or falling down. If we also consider that specialized workforce has been rapidly ageing, there is a clear opportunity for the use of SRLs as assistive tools to reduce fatigue and increase safety [102]. The building construction industry presents a similar opportunity, being an application area where many occupational injuries are caused by falls [103].

Other envisaged applications of supernumerary arms include performing tasks requiring three hands, e.g. welding [35] (figure 7(b)), and executing parallel tasks with the human, e.g. fruit picking [31] (figure $7(\mathrm{c})$ ). In the first case, particularly dexterous structures are needed, as for example the limbs presented in $[34,35]$, whereas in the second strength and wearability are the most important features. To attain them both, Veronneau et al [31] designed a powerful arm with a remote actuation system.

SRLs are potentially useful in tasks where external perception is needed, such as opening a door while the natural hands and arms are holding an object. A human-in-the-loop control could be used in this case, leveraging the human's superior perception system to help the SRLs. Guggenheim et al [86] studied the effects of controlling SRLs together with natural limbs in manipulation tasks. SRLs that are attached directly to the human body transmit forces from the environment to it. Guggenheim et al [83] also investigated this inherent haptic feedback and how it allows the human to perceive the interaction between the robot and the environment, monitor its actions, and effectively control the robot.

Another area in which SRLs, and in particular supernumerary legs, could be useful is mobility assistance for the elderly. For older adults, the risk of losing balance and falling down is high [104]. Loss of mobility for the elderly also includes difficulties in safely sitting down or standing up [105]. Standard tools used to address these problems present significant limitations, and are not suitable for the active lifestyle that senior 


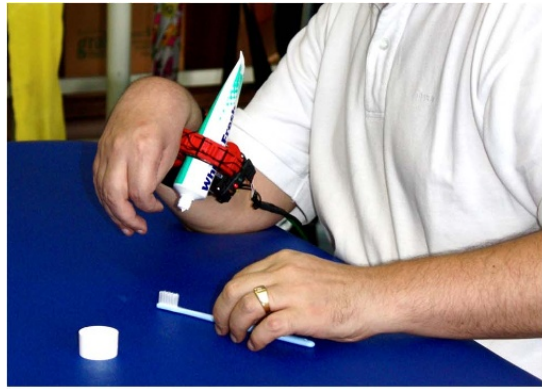

(a)

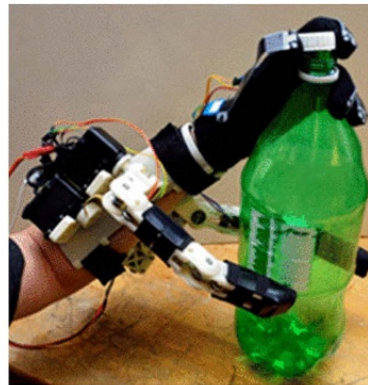

(b)

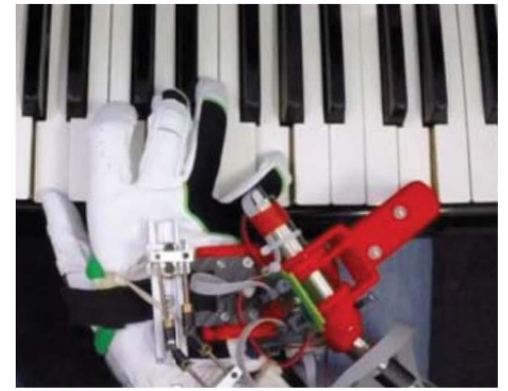

(c)

Figure 8. Applications of supernumerary robotic fingers. (a) Finger worn on a paretic limb of a stroke patient to compensate for missing grasping abilities and perform bimanual tasks [53]. (b), (c) Fingers worn on healthy hands to augment dexterity ((b) (c) [2015] IEEE. Reprinted, with permission, from [71]; (c) (c) [2018] IEEE. Reprinted, with permission, from [57]).

citizens expect in the future modern societies. Aids such as canes and braces require the use of one or both arms to be functional, and significant forces must be exerted in order to avoid slips. Robotic walkers are easier to use but they work only on smooth surfaces, mainly inside the home since stairs could represent an insurmountable stumbling block. Conversely, a SRL could be used in this context as an assistive tool that provides balance aid and weight compensation while leaving the user's arms free, as proposed in [106]. The SRL could also assist elderly when sitting down or standing up, or as an emergency help in case of slips. Being wearable and not based on wheels, the SRL is able to follow and help users in any situation, including stairs and irregular terrain (streets, parks, etc).

The fields of assistive and rehabilitation robotics represent a new and promising research directions for supernumerary arms and legs. There is a large number of impaired people who need mobility assistance as a consequence of legs impairments, spinal injuries or neurological disorders. SRLs can be used to assist these patients without requiring any arm effort, and leaving their legs free to move and exercise.

Most of the studies analyzed in this paper focus on how additional arms and legs can be used to support humans in performing physical tasks. Supernumerary limbs, however, can have also other uses. In [29], for example, a SRL working as a wearable haptic device was presented. Another application of SRLs is communication [107]. The device proposed in [108], for instance, was used both as a physical support and as a tool for communicating emotions. In [109, 110], an artistic performance based on supernumerary arms, entitled Mutation, was presented.

\subsection{Applications for fingers}

Supernumerary robotic fingers have been successfully exploited for both assistive and augmentative applications. Regarding the first, they can be worn on the paretic upper limb of patients with motor disabilities to compensate for their lost manipulative skills. The use of supernumerary fingers as compensatory tools, not only allows patients to regain independence in daily activities, but also opens a conceptually new rehabilitative scenario, as wearing a robotic aid may promote the re-use of the paretic limb. One of the main categories of patients that may benefit from the adoption of supernumerary fingers is that of stroke patients with a paretic upper limb [53] (figure 8(a)).

Following a stroke, the gold standard of traditional rehabilitation of a hand motor paresis, when the deficit is stabilized, is mainly based on ergotherapy [111] or on constraint-induced movement therapy (CIMT). The latter requires the prolonged immobilization of the healthy hand to promote the re-use of the affected one [112]. Ergotherapy consists of teaching alternative strategies for functional compensation (rather than re-learning the lost function), often with the help of dedicated aids to be used with the unaffected limb. This approach is neither ergonomic nor natural, carrying the risk of even increasing pathological motor patterns, for instance, by worsening tonic flexion at the forearm of the paretic limb [113]. Immobilization, even in healthy subjects, may produce shrinking of the cortical representation of the immobilized muscles and worsening of some kinematic parameters of a reaching-to-grasp action $[114,115]$. Thus, both ergotherapy and CIMT are not satisfactory in terms of re-acquisition of bimanual tasks, and carry the ultimate risk of increasing the functional disparities between the two upper limbs.

The adoption of a supernumerary finger can be considered a truly conceptual advance in the context of traditional rehabilitation approaches, as it represents an active and motivational assistance device. Patients that could benefit more from a supernumerary finger are those that are affected by a hand paresis, but still have preserved muscular function in the proximal segment of the arm/shoulder, a condition that allows the 
patient to counteract gravity and move the arm upwards. Preliminary results showed that chronic stroke patients with residual arm mobility are able to intuitively recover unimanual and bimanual grasping abilities in activities of daily living (ADLs) by using a supernumerary finger [63]. In addition, they had an enthusiastic feeling towards the robotic aid.

To compensate for missing grasping capabilities in patients with more severe conditions, it is possible to couple supernumerary fingers or hands with gravity support systems, as proposed in $[80,116]$.

As envisaged in [70], supernumerary robotic fingers could also be used for practicing intense movement training in a domestic context, which is still an unsolved challenge in rehabilitation and assistive engineering [117]. Rehabilitation with supernumerary robotic fingers is still in its infancy, and comparative trials with traditional approaches have to be implemented.

Soft and underactuated devices are particularly suited for assistive scenarios as they are robust to impacts and easy to command. Also control and feedback interfaces should be tailored to the specific application. The wearable EMG-based interface presented in [53], for example, detects the activation of the frontalis muscle that has a bilateral cortical representation and thus is spared in case of a motor stroke, either in the left or in the right hemisphere.

Supernumerary fingers with multiple actuated DoFs have applications in augmentative scenarios. One of the most studied ones is the manipulation with a single hand of objects whose size would require two hands (figure 8(b)). In this view, different solutions have been explored using one or more fingers [43, 71, 118]. Another use of supernumerary fingers is the augmentation of the hand dexterity (figure $8(\mathrm{c})$ ). For instance, they can help in playing piano with different chords [58], or can be exploited for in hand manipulation of objects [45] or to hold an object while the hand manipulates it [71].

\subsection{Concluding remarks}

From the analysis of the literature, it emerges that although there is an increasing interest in SRLs, the related research area is still young and the applicability of these technologies in real-world scenarios is still limited. Most of the supernumerary limbs that have been revised in this paper are prototypes and have a quite low technology readiness level (TRL), i.e. TRL $<4$.

Higher levels of TRL have been reached by some models of robotic fingers, as for instance in papers where they have been tested with patients in ADL (TRL 5) [63]. In addition, there are examples in which the design of supernumerary fingers has reached TRL 7, e.g. the Third Thumb developed by Dani Clode in collaboration with the University College London [65], and even TRL 8 (pre-commercialization phase), e.g. Sixto, by the Italian start-up Existo ${ }^{5}$.

The success of supernumerary robotic arms and legs critically depends on the satisfaction of several design constraints. These are similar to those necessary to build supernumerary fingers (wearability, lightness, safety, etc), but are more stringent and more difficult to meet with structures that must be large and powerful. This is why in most of the articles, only the design of an initial prototype is presented, with preliminary laboratory evaluations.

SRLs have the potential to constitute a technological revolution, allowing for innovative applications in several fields. However, examples of an effective use of SRLs outside research labs are still limited. The risk is to develop technologically advanced devices that have limited uptake despite their robust and sound engineering. Traditionally, users have been actively involved in pre-development phases, i.e. in requirements engineering and in prototyping and testing activities. With the new areas of deployment of SRLs, there is a need to involve users in the early stages of development and continuously improve the quality and adapt the features of the devices based on their feedback and understandings. In order for SRLs to become widely deployed, there is a need to keep the users at the center of all the development process, adopting a user-centric and iterative approach [119].

\section{Perspectives}

'Anaxagoras says that humans are the most intelligent of the animals because of their hands' (De partibus animalium, Aristotle). The intuition of the philosopher clearly states the central role of bodily skills for the cognitive evolution of humans. Recently, evolutionary scientists have studied the bodily basis of thoughts arguing that humans do not simply inhabit their bodies but literally use them to think [120, 121]. Today the centrality of the sensorimotor bodily skills in humans has been largely overshadowed by the technological augmentation of cognitive abilities. Think of cognitive augmentation given by smartphones [122], by novel BMIs, such as the one proposed by E. Musk's company Neuralink [123], or by non invasive brain stimulation techniques [124]. Relegating the augmentation of bodily intelligence to a secondary role might unbalance

5 Sixto: https://e-novia.it/startup/existo/. 
cognitive and bodily skills development of our species. SRLs long term objective may be the augmentation of human bodily intelligence, i.e. the enhancement of human sensorimotor capabilities of bodily interaction with the environment.

As it emerges from the literature review, most of the success or the failure of this vision depends on the quality of the bilateral sensorimotor interface between the human body (and brain) and the artificial limbs (see section 3.3). The development of intelligent and non-invasive interfaces could enable a completely novel and interesting scenario where also non wearable robots, e.g. robotic arms, may be perceived as an extension of the human body. This possibility would solve many of the design issues faced by wearable devices (see section 2.3) and open to many other application fields.

The paradigm shift would consist in decoupling the flow of the sensorimotor information (provided by the sensorimotor interface), from the action of motor augmentation (performed by the supernumerary limbs). While, in our vision, motor augmentation can even occur far from the human body (e.g. with a grounded robot), the interface is mandatorily worn by the user. The role of the sensorimotor interface is to provide motor commands from the human to the SRL, and to provide feedback (mostly haptic) from the SRL to the human, as also pointed out in [8].

A promising direction for the control of SRLs is to exploit users' musculoskeletal redundancy, i.e. body motions and muscle activations that do not affect the main physical interaction with the environment performed by biological hands [8]. In this way, a true bodily augmentation would be achieved and users would be able to simultaneously and concurrently control natural and artificial limbs. The concept of musculoskeletal redundancy has been applied by d'Avella et al for muscle-to-force mapping in virtual surgery [89], and by Wu and Asada to control SRLs through the kinematic redundancy of the human elbow [71].

The sensory part of the augmentation consists in displaying, through a wearable device, haptic stimuli providing information about the robotic limb and the task, including cues on robot constraints, obstacles, and grasp quality. The placement of feedback interfaces and the type of transmitted stimuli should be carefully chosen, aiming at compensating for the missing direct physical connection with the SRL. The choice of the tactile channel allows to maintain the main physiological channel of sensorimotor feedback $[8,125]$ and to avoid overloading of vision and/or hearing. Dually with respect to the motor control, haptic information must not interfere with other haptic stimuli coming from the interaction of natural limbs with the environment, otherwise it could be difficult for the user to detect and interpret it. In [126], authors present preliminary, but promising results on the use of vibrotactile feedback to convey information on the state of a collaborative robot in absence of auditory and visual cues.

The idea that we propose is to close the loop between the SRL and the human somatosensory system, allowing users to control the robot in a way that is truly independent from their natural movements, but can be coordinated with them. Methods to assess human ability in controlling and perceiving supernumerary degrees of freedom need to be developed.

The possibility of having supernumerary limbs not only worn on the user's body, but also distant from the person, also requires to rethink robot planning and control [127, 128]. Thanks to SRLs, humans will be able to perform augmented manipulation tasks, i.e. manipulation tasks requiring at least one supernumerary limb in addition to biological limbs to be accomplished. Contrary to a collaborative scenario, robot actions are not executed autonomously but are partially commanded by the human. This requires a seamless interplay between the bodily intelligence of the human and the artificial intelligence of the SRL. A supervisory control system constituting the robot artificial intelligence must be in charge of dynamically assigning roles to humans and robots, based on both agents' capabilities and availability, and on users' preferences. A functional trade-off between the degree of autonomy of the robotic limb and its direct control by the human must be found.

The consideration of non-wearable SRLs also opens the study of what are the neuroscientific implications in the perception of peripersonal space, body representation, sense of agency and, possibly, embodiment when using robotic devices which are physically detached from the user but connected to her/his body through a wearable sensorimotor interface. Current literature on these topics mostly considers wearable fingers.

We think that focusing on the user centered design of sensorimotor interfaces and of new methodologies for controlling SRLs are the necessary steps to boost the field of robotic human augmentation. In addition, the development of a new generation of wearable, unobtrusive sensorimotor interfaces that can be freely and easily worn/unworn could impact not only the field of human-robot interaction, but also other areas where the integration of human and artificial intelligence are important, including augmented and virtual reality applications, home automation, and autonomous vehicles.

As the most promising current uses of SRLs are in the medical field (see section 5), bodily augmentation by SRLs is expected to have a relevant impact on assistive robotics in the near future. Applications in other 
fields are already being explored and can only be limited by our imagination. The world, as we know it, is designed for people with two arms, two legs, and ten fingers, so we might not be immediately ready to find examples where supernumerary limbs are truly necessary outside the healthcare domain. However, that is just because, so far, research on SRLs has mainly focused on their design and low-level control. There is the need to extensively test these devices in real-world contexts to better appreciate their potentialities.

\section{Data availability statement}

No new data were created or analyzed in this study.

\section{ORCID iDs}

Domenico Prattichizzo (1) https://orcid.org/0000-0001-9051-9698

Maria Pozzi ำ https://orcid.org/0000-0002-2390-1907

Tommaso Lisini Baldi (1) https://orcid.org/0000-0002-2930-955X

Monica Malvezzi (i) https://orcid.org/0000-0002-2158-5920

Irfan Hussain (1) https://orcid.org/0000-0003-2759-0306

Simone Rossi (1) https://orcid.org/0000-0001-6697-9459

Gionata Salvietti 구 https://orcid.org/0000-0001-9170-4051

\section{References}

[1] Cinel C, Valeriani D and Poli R 2019 Neurotechnologies for human cognitive augmentation: current state of the art and future prospects Front. Hum. Neurosci. 1313

[2] Makin T R, de Vignemont F and Faisal A A 2017 Neurocognitive barriers to the embodiment of technology Nat. Biomed. Eng. $11-3$

[3] Kieliba P, Clode D, Maimon-Mor R O and Makin T R 2021 Robotic hand augmentation drives changes in neural body representation Sci. Robot. 654

[4] Herr H Robotics for human augmentation (available at: www.sciencemag.org/journals/robotics/human-augmentation) (Accessed May 2021)

[5] Masia L et al 2018 Soft wearable assistive robotics: exosuits and supernumerary limbs Wearable Exoskeleton Systems: Design, Control and Applications (Control, Robotics and Sensors) (Institution of Engineering and Technology) pp 219-54

[6] Tong Y and Liu J 2021 Review of research and development of supernumerary robotic limbs IEEE CAA J. Autom. Sin. $8929-52$

[7] Yang B, Huang J, Chen X, Xiong C and Hasegawa Y 2021 Supernumerary robotic limbs: a review and future outlook IEEE Trans. Med. Robot. Bionics 3 623-39

[8] Dominijanni G et al 2021 Enhancing human bodies with extra robotic arms and fingers: the neural resource allocation problem Nat. Mach. Intell. (arXiv:2103.17252v1)

[9] Eldershaw C and Duff D 2007 Portable personal wearable active third arm US Patent App. 11/210,841

[10] Davenport C, Parietti F and Asada H H 2012 Design and biomechanical analysis of supernumerary robotic limbs Dynamic Systems and Conf vol 45295 (American Society of Mechanical Engineers) pp 787-93

[11] Llorens-Bonilla B, Parietti F and Asada H H 2012 Demonstration-based control of supernumerary robotic limbs Proc. IEEE/RSJ Int. Conf. Intelligent Robots and Systems, IROS pp 3936-42

[12] Parietti F and Asada H H 2013 Dynamic analysis and state estimation for wearable robotic limbs subject to human-induced disturbances 2013 IEEE Int. Conf. Robotics and Automation (IEEE) pp 3880-7

[13] Parietti F, Chan K and Asada H 2014 Bracing the human body with supernumerary robotic limbs for physical assistance and load reduction Proc. IEEE Int. Conf. Robotics and Automation, ICRA pp 141-8

[14] Parietti F and Asada H H 2014 Supernumerary robotic limbs for aircraft fuselage assembly: body stabilization and guidance by bracing Proc. IEEE Int. Conf. Robotics and Automation, ICRA pp 1176-83

[15] Llorens-Bonilla B and Asada H H 2013 Control and coordination of supernumerary robotic limbs based on human motion detection and task petri net model Dynamic Systems and Conf. vol 56130 (American Society of Mechanical Engineers) p V002T27A006

[16] Bonilla B L and Asada H H 2014 A robot on the shoulder: coordinated human-wearable robot control using coloured petri nets and partial least squares predictions 2014 IEEE Int. Conf. Robotics and Automation (ICRA) (IEEE) pp 119-25

[17] Bright Z and Asada H H 2017 Supernumerary robotic limbs for human augmentation in overhead assembly tasks Proc. Robotics: Science and Systems (Cambridge, Massachusetts)

[18] Nguyen P H, Mohd I I, Sparks C, Arellano F L, Zhang W and Polygerinos P 2019 Fabric soft poly-limbs for physical assistance of daily living tasks 2019 Int. Conf. Robotics and Automation (ICRA) (IEEE) pp 8429-35

[19] Parietti F and Asada H H 2017 Independent, voluntary control of extra robotic limbs 2017 IEEE Int. Conf. Robotics and Automation (ICRA) (IEEE) pp 5954-61

[20] Khazoom C, Caillouette P, Girard A and Plante J-S 2020 A supernumerary robotic leg powered by magnetorheological actuators to assist human locomotion IEEE Robot. Autom. Lett. 5 5143-50

[21] Xu C, Liu Y and Li Z 2019 Biomechtronic design of a supernumerary robotic limbs for industrial assembly 2019 IEEE 4th Int. Conf. Advanced Robotics and Mechatronics (ICARM) (IEEE) pp 553-8

[22] Guggenheim J, Hoffman R, Song H and Asada H H 2020 Leveraging the human operator in the design and control of supernumerary robotic limbs IEEE Robot. Autom. Lett. 5 2177-84

[23] Khodambashi R, Weinberg G, Singhose W, Rishmawi S, Murali V and Kim E 2016 User oriented assessment of vibration suppression by command shaping in a supernumerary wearable robotic arm 2016 IEEE-RAS 16th Int. Conf. Humanoid Robots (Humanoids) (IEEE) pp 1067-72 
[24] Kojima A, Yamazoe H, Chung M G and Lee J-H 2017 Control of wearable robot arm with hybrid actuation system 2017 IEEE/SICE Int. Symp. System Integration (SII) (IEEE) pp 1022-7

[25] Penaloza C, Hernandez-Carmona D and Nishio S 2018 Towards intelligent brain-controlled body augmentation robotic limbs 2018 IEEE Int. Conf. Systems, Man and Cybernetics (SMC) (IEEE) pp 1011-15

[26] Penaloza C I and Nishio S 2018 Bmi control of a third arm for multitasking Sci. Robot. 3 eaat1228

[27] Vatsal V and Hoffman G 2018 Design and analysis of a wearable robotic forearm 2018 IEEE Int. Conf. Robotics and Automation (ICRA) (IEEE) pp 1-8

[28] Al-Sada M, Höglund T, Khamis M, Urbani J and Nakajima T 2019 Orochi: investigating requirements and expectations for multipurpose daily used supernumerary robotic limbs Proc. 10th Augmented Human Int. Conf. 2019 pp 1-9

[29] Al-Sada M, Jiang K, Ranade S, Kalkattawi M and Nakajima T 2019 HapticSnakes: multi-haptic feedback wearable robots for immersive virtual reality Virtual Reality 24 191-209

[30] Véronneau C, Denis J, Lebel L-P, Denninger M, Plante J-S and Girard A 2019 A lightweight force-controllable wearable arm based on magnetorheological-hydrostatic actuators 2019 Int. Conf. Robotics and Automation (ICRA) (IEEE) pp 4018-24

[31] Véronneau C, Denis J, Lebel L-P, Denninger M, Blanchard V, Girard A and Plante J-S 2020 Multifunctional remotely actuated 3-dof supernumerary robotic arm based on magnetorheological clutches and hydrostatic transmission lines IEEE Robot. Autom. Lett. 5 2546-53

[32] Zhang Q, Zhu Y, Zhao X, Yang Y, Jing H, Zhang G and Zhao J 2020 Design of reconfigurable supernumerary robotic limb based on differential actuated joints Int. J. Comput. Inf. Eng. 14 115-22 (available at: https://publications.waset.org/10011167/design-ofreconfigurable-supernumerary-robotic-limb-based-on-differential-actuated-joints)

[33] Kurek D A and Asada H H 2017 The mantisbot: design and impedance control of supernumerary robotic limbs for near-ground work 2017 IEEE Int. Conf. Robotics and Automation (ICRA) (IEEE) pp 5942-7

[34] Saraiji M Y, Sasaki T, Kunze K, Minamizawa K and Inami M 2018 Metaarms: body remapping using feet-controlled artificial arms Proc. 31st Annual Symp. User Interface Software and Technology pp 65-74

[35] Sasaki T, Saraiji M Y, Fernando C L, Minamizawa K and Inami M 2017 Metalimbs: multiple arms interaction metamorphism ACM SIGGRAPH 2017 Emerging Technologies pp 1-2

[36] Ciullo A S, Catalano M G, Bicchi A and Ajoudani A 2018 A supernumerary soft robotic hand-arm system for improving worker ergonomics Int. Symp. Wearable Robotics (Springer) pp 520-4

[37] Daniel P H and Asada H H 2020 Stable crawling policy for wearable superlimbs attached to a human with tuned impedance 2020 IEEE/RSJ Int. Conf. Intelligent Robots and Systems (IROS) pp 3496-503

[38] Liang X, Yap H K, Guo J, Yeow R C H, Sun Y and Chui C K 2017 Design and characterization of a novel fabric-based robotic arm for future wearable robot application 2017 IEEE Int. Conf. Robotics and Biomimetics (ROBIO) (IEEE) pp 367-72

[39] Nguyen P H, Sparks C, Nuthi S G, Vale N M and Polygerinos P 2019 Soft poly-limbs: toward a new paradigm of mobile manipulation for daily living tasks Soft Robot. $638-53$

[40] Parietti F, Chan K C, Hunter B and Asada H H 2015 Design and control of supernumerary robotic limbs for balance augmentation 2015 IEEE Int. Conf. Robotics and Automation (ICRA) (IEEE) pp 5010-17

[41] Parietti F and Asada H 2016 Supernumerary robotic limbs for human body support IEEE Trans. Robot. 32 301-11

[42] Gonzalez D J and Asada H H 2018 Design of extra robotic legs for augmenting human payload capabilities by exploiting singularity and torque redistribution 2018 IEEE/RSJ Int. Conf. Intelligent Robots and Systems (IROS) (IEEE) pp 4348-54

[43] Prattichizzo D, Salvietti G, Chinello F and Malvezzi M 2014 An object-based mapping algorithm to control wearable robotic extra-fingers 2014 IEEE/ASME Int. Conf. Advanced Intelligent Mechatronics (IEEE) pp 1563-8

[44] Hussain I, Santarnecchi E, Leo A, Ricciardi E, Rossi S and Prattichizzo D 2017 A magnetic compatible supernumerary robotic finger for functional magnetic resonance imaging (fmri) acquisitions: device description and preliminary results 2017 Int. Conf. Rehabilitation Robotics (ICORR) (IEEE) pp 1177-82

[45] Prattichizzo D, Malvezzi M, Hussain I and Salvietti G 2014 The sixth-finger: a modular extra-finger to enhance human hand capabilities 23rd IEEE Int. Symp. Robot and Human Interactive Communication (IEEE) pp 993-8

[46] Hussain I, Meli L, Pacchierotti C, Salvietti G and Prattichizzo D 2015 Vibrotactile haptic feedback for intuitive control of robotic extra fingers 2015 IEEE World Haptics Conference (WHC) pp 394-9

[47] Hussain I, Salvietti G, Meli L, Pacchierotti C, Cioncoloni D, Rossi S and Prattichizzo D 2015 Using the robotic sixth finger and vibrotactile feedback for grasp compensation in chronic stroke patients 2015 IEEE Int. Conf. Rehabilitation Robotics (ICORR) (IEEE) pp 67-72

[48] Hussain I, Spagnoletti G, Salvietti G and Prattichizzo D 2016 An emg interface for the control of motion and compliance of a supernumerary robotic finger Front. Neurorobot. 1018

[49] Sobajima M, Sato Y, Xufeng W and Hasegawa Y 2015 Improvement of operability of extra robotic thumb using tactile feedback by electrical stimulation 2015 Int. Symp. Micro-NanoMechatronics and Human Science (MHS) (IEEE) pp 1-3

[50] Meraz N S, Shikida H and Hasegawa Y 2017 Auricularis muscles based control interface for robotic extra thumb 2017 Int. Symp. Micro-NanoMechatronics and Human Science (MHS) (IEEE) pp 1-3

[51] Shikida H, Noel S-M and Hasegawa Y 2017 Somatosensory feedback improves operability of extra robotic thumb controlled by vestigial muscles 2017 Int. Symp. Micro-NanoMechatronics and Human Science (MHS) (IEEE) pp 1-4

[52] Zhu Y, Ito T, Aoyama T and Hasegawa Y 2019 Development of sense of self-location based on somatosensory feedback from finger tips for extra robotic thumb control Robomech J. 6 1-10

[53] Hussain I, Salvietti G, Spagnoletti G and Prattichizzo D 2016 The soft-sixthfinger: a wearable EMG controlled robotic extra-finger for Grasp compensation in chronic stroke patients IEEE Robot. Autom. Lett. 1 1000-6

[54] Franco L, Salvietti G and Prattichizzo D 2019 Command acknowledge through tactile feedback improves the usability of an emg-based interface for the frontalis muscle 2019 IEEE World Conf. (WHC) (IEEE) pp 574-9

[55] Hussain I, Meli L, Pacchierotti C and Prattichizzo D 2017 A soft robotic supernumerary finger and a wearable cutaneous finger interface to compensate the missing grasping capabilities in chronic stroke patients 2017 IEEE World Conf. (WHC) (IEEE) pp 183-8

[56] Leigh S-w, Denton T, Parekh K, Peebles W, Johnson M and Maes P 2018 Morphology extension kit: a modular robotic platform for physically reconfigurable wearables Proc. Twelfth Int. Conf. Tangible, Embedded and Embodied Interaction pp 11-18

[57] Cunningham J, Hapsari A, Guilleminot P, Shafti A and Faisal A A 2018 The supernumerary robotic 3rd thumb for skilled music tasks 2018 7th IEEE Int. Conf. on Biomedical Robotics and Biomechatronics (Biorob) (IEEE) pp 665-70

[58] Shafti A, Haar S, Zaldivar R M, Guilleminot P and Faisal A A 2021 Playing the piano with a robotic third thumb: Assessing constraints of human augmentation bioRxiv (https://doi.org/10.1101/2020.05.21.108407) 
[59] Hussain I, Anwar M, Iqbal Z, Muthusamy R, Malvezzi M, Seneviratne L, Gan D, Renda F and Prattichizzo D 2019 Design and prototype of supernumerary robotic finger (srf) inspired by fin ray ${ }^{\circledR}$ effect for patients suffering from sensorimotor hand impairment 2019 2nd IEEE Int. Conf. Soft Robotics (RoboSoft) (IEEE) pp 398-403

[60] Singh K S and Thondiyath A 2019 Design and analysis of a soft bidirectional bending actuator for human-robot interaction applications 2019 28th IEEE Int. Conf. Robot and Human Interactive Communication (RO-MAN) (IEEE) pp 1-6

[61] Gerez L, Gao G, Dwivedi A and Liarokapis M 2020 A hybrid, wearable exoskeleton glove equipped with variable stiffness joints, abduction capabilities and a telescopic thumb IEEE Access 8 173345-58

[62] Hussain I, Salvietti G, Malvezzi M and Prattichizzo D 2015 Design guidelines for a wearable robotic extra-finger 2015 IEEE 1st Int. Forum on Research and Technologies for Society and Industry Leveraging a Better Tomorrow (RTSI) (IEEE) pp 54-60

[63] Salvietti G, Hussain I, Cioncoloni D, Taddei S, Rossi S and Prattichizzo D 2017 Compensating hand function in chronic stroke patients through the robotic sixth finger IEEE Trans. Neural Syst. Rehabil. Eng. 25 142-50

[64] Hussain I and Prattichizzo D 2020 Augmenting Human Manipulation Abilities With Supernumerary Robotic Limbs (Berlin: Springer)

[65] Clode D Third thumb (available at: www.daniclodedesign.com/thethirdthumb) (Accessed May 2021)

[66] Agharese N, Cloyd T, Blumenschein L H, Raitor M, Hawkes E W, Culbertson H and Okamura A M 2018 Hapwrap: soft growing wearable haptic device 2018 IEEE Int. Conf. Robotics and Automation (ICRA) (IEEE) pp 1-5

[67] Wu F and Asada H 2014 Bio-artificial synergies for grasp posture control of supernumerary robotic fingers Proc. Robotics: Science and Systems (Berkeley, USA)

[68] Wu F Y and Asada H H 2018 Decoupled motion control of wearable robot for rejecting human induced disturbances 2018 IEEE Int. Conf. Robotics and Automation (ICRA) (IEEE) pp 1-8

[69] Wu F Y and Asada H H 2016 Implicit and intuitive grasp posture control for wearable robotic fingers: a data-driven method using partial least squares IEEE Trans. Robot. 32 176-86

[70] Ort T, Wu F, Hensel N C and Asada H H 2015 Supernumerary robotic fingers as a therapeutic device for hemiparetic patients Dynamic Systems and Conf. vol 57250 (American Society of Mechanical Engineers) p V002T27A010

[71] Wu F Y and Asada H H 2015 "Hold-and-manipulate" with a single hand being assisted by wearable extra fingers 2015 IEEE Int. Conf. Robotics and Automation (ICRA) (IEEE) pp 6205-12

[72] Tiziani L, Hart A, Cahoon T, Wu F, Asada H H and Hammond F L 2017 Empirical characterization of modular variable stiffness inflatable structures for supernumerary grasp-assist devices Int. J. Robot. Res. 36 1391-1413

[73] Hammond III F L, Wu F and Asada H H 2018 Variable stiffness pneumatic structures for wearable supernumerary robotic devices Robotics Research (Berlin: Springer) pp 201-17

[74] Hussain I, Spagnoletti G, Salvietti G and Prattichizzo D 2017 Toward wearable supernumerary robotic fingers to compensate missing grasping abilities in hemiparetic upper limb Int. J. Robot. Res. 36 1414-36

[75] Malvezzi M, Iqbal Z, Valigi M C, Pozzi M, Prattichizzo D and Salvietti G 2019 Design of multiple wearable robotic extra fingers for human hand augmentation Robotics 8102

[76] Liu S, Zhu Y, Zhang Z, Fang Z, Tan J, Peng J, Song C, Asada H and Wang Z 2020 Otariidae-inspired soft-robotic supernumerary flippers by fabric kirigami and origami IEEE/ASME Trans. Mechatron. (https://doi.org/10.1109/TMECH.2020.3045476)

[77] Zhou J, Chen X, Chang U, Liu Y, Chen Y and Wang Z 2019 A grasping component mapping approach for soft robotic end-effector control 2019 2nd IEEE Int. Conf. Soft Robotics (RoboSoft) (IEEE) pp 650-5

[78] Ciullo A S, Felici F, Catalano M G, Grioli G, Ajoudani A and Bicchi A 2018 Analytical and experimental analysis for position optimization of a grasp assistance supernumerary robotic hand IEEE Robot. Automat. Lett. 3 4305-12

[79] Catalano M G, Grioli G, Farnioli E, Serio A, Piazza C and Bicchi A 2014 Adaptive synergies for the design and control of the PISA/IIT softhand Int. J. Robot. Res. 33 768-82

[80] Ciullo A S et al 2020 A novel soft robotic supernumerary hand for severely affected stroke patients IEEE Trans. Neural Syst. Rehabil. Eng. 28 1168-77

[81] Walsh C 2018 Human-in-the-loop development of soft wearable robots Nat. Rev. Mater. $378-80$

[82] Malvezzi M, Valigi M C, Salvietti G, Iqbal Z, Hussain I and Prattichizzo D 2018 Design criteria for wearable robotic extra-fingers with underactuated modular structure Int. Conf. IFToMM ITALY (Springer) pp 509-17

[83] Guggenheim J W and Asada H H 2020 Inherent haptic feedback from supernumerary robotic limbs IEEE Trans. Haptics $14123-31$

[84] Dougherty Z and Winck R C 2019 Evaluating the performance of foot control of a supernumerary robotic limb Dynamic Systems and Conf. vol 59162 (American Society of Mechanical Engineers) p V003T16A003

[85] Kelley J F 1984 An iterative design methodology for user-friendly natural language office information applications ACM Trans. Inf. Syst. (TOIS) 2 26-41

[86] Guggenheim J W, Parietti F, Flash T and Asada H H 2020 Laying the groundwork for intra-robotic-natural limb coordination: is fully manual control viable? ACM Trans. Hum.-Robot Interact. 9 1-12

[87] Casalino A, Messeri C, Pozzi M, Zanchettin A M, Rocco P and Prattichizzo D 2018 Operator awareness in human-robot collaboration through wearable vibrotactile feedback IEEE Robot. Automat. Lett. 3 4289-96

[88] Mehring C et al 2019 Augmented manipulation ability in humans with six-fingered hands Nat. Commun. 10 1-9

[89] d'Avella A and Lacquaniti F 2013 Control of reaching movements by muscle synergy combinations Front. Computat. Neurosci. 742

[90] Santello M et al 2016 Hand synergies: integration of robotics and neuroscience for understanding the control of biological and artificial hands Phys. Life Rev. 17 1-23

[91] Rossi S et al 2021 Emerging of new bioartificial corticospinal motor synergies using a robotic additional thumb Sci. Rep. in press

[92] Hallett M 2007 Transcranial magnetic stimulation: a primer Neuron 55 187-99

[93] Rossi S and Rossini P M 2004 Tms in cognitive plasticity and the potential for rehabilitation Trends Cogn. Sci. $8273-9$

[94] Di Pino G, Maravita A, Zollo L, Guglielmelli E and Di Lazzaro V 2014 Augmentation-related brain plasticity Front. Syst. Neurosci. 8109

[95] Abdi E, Burdet E, Bouri M, Himidan S and Bleuler H 2016 In a demanding task, three-handed manipulation is preferred to two-handed manipulation Sci. Rep. 621758

[96] Holmes N P and Spence C 2004 The body schema and multisensory representation (s) of peripersonal space Cogn. Process. 5 94-105

[97] Shokur S, O’Doherty J E, Winans J A, Bleuler H, Lebedev M A and Nicolelis M A 2013 Expanding the primate body schema in sensorimotor cortex by virtual touches of an avatar Proc. Natl Acad. Sci. 110 15121-6 
[98] Meraz N S, Sobajima M, Aoyama T and Hasegawa Y 2018 Modification of body schema by use of extra robotic thumb Robomech J. 53

[99] Hoyet L, Argelaguet F, Nicole C and Lécuyer A 2016 Wow! “I have six fingers!”: would you accept structural changes of your hand in VR? Front. Robot. AI 327

[100] Fröhner J, Salvietti G, Beckerle P and Prattichizzo D 2019 Can wearable haptic devices foster the embodiment of virtual limbs? IEEE Trans. Haptics 12 339-49

[101] Shin C-Y, Bae J and Hong D 2015 Ceiling work scenario based hardware design and control algorithm of supernumerary robotic limbs 2015 15th Int. Conf. Control, Automation and Systems (ICCAS) (IEEE) pp 1228-30

[102] Mosisa A and Hipple S 2006 Trends in labor force participation in the United States Monthly Lab. Rev. 12935

[103] Statistics, Bureau of Labor 2016 Census of Fatal Occupational Injuries (Washington, DC: Bureau of Labor Statistics)

[104] Winter D A 1995 Human balance and posture control during standing and walking Gait Posture 3 193-214

[105] Mourey F, Pozzo T, Rouhier-Marcer I and Dider J-P 1998 A kinematic comparison between elderly and young subjects standing up from and sitting down in a chair Age Ageing 27 137-46

[106] Wu X, Liu H, Liu Z, Chen M, Wan F, Fu C, Asada H, Wang Z and Song C 2020 Robotic cane as a soft superlimb for elderly sit-to-stand assistance 2020 3rd IEEE Int. Conf. on Soft Robotics (RoboSoft) (IEEE) pp 599-606

[107] Tran A, Somanath S and Sharlin E 2018 Supernumerary arms for gestural communication Extended Abstracts of the 2018 Conf. Human Factors in Computing Systems pp 1-6

[108] Xie H, Mitsuhashi K and Torii T 2019 Augmenting human with a tail Proc. 10th Augmented Human Int. Conf. 2019 pp 1-7

[109] Hammad N, Sanoubari E, Finn P, Somanath S, Young J E and Sharlin E 2019 Mutation: leveraging performing arts practices in cyborg transitioning Proc. 2019 on Creativity and Cognition pp 53-59

[110] Hammad N, Somanath S, Sharlin E and Finn P 2019 Exploring the experience of becoming and unbecoming a cyborg using performing arts techniques Tech. Rep. (University of Calgary, Science Department)

[111] Gillen G 2015 Stroke Rehabilitation: A Function-Based Approach (Elsevier Health Sciences)

[112] Taub E, Miller N, Novack T, Cook 3rd E, Fleming W, Nepomuceno C, Connell J and Crago J 1993 Technique to improve chronic motor deficit after stroke Arch. Phys. Med. Rehabil. 74 347-54

[113] Michaelsen S M, Jacobs S, Roby-Brami A and Levin M F 2004 Compensation for distal impairments of grasping in adults with hemiparesis Exp. Brain Res. 157 162-73

[114] Bassolino M, Bove M, Jacono M, Fadiga L and Pozzo T 2012 Functional effect of short-term immobilization: kinematic changes and recovery on reaching-to-grasp Neuroscience 215 127-34

[115] Bassolino M, Campanella M, Bove M, Pozzo T and Fadiga L 2014 Training the motor cortex by observing the actions of others during immobilization Cerebral Cortex 24 3268-76

[116] Hussain I, Salvietti G, Spagnoletti G, Malvezzi M, Cioncoloni D, Rossi S and Prattichizzo D 2017 A soft supernumerary robotic finger and mobile arm support for grasping compensation and hemiparetic upper limb rehabilitation Robot. Auton. Syst. 93 1-12

[117] Varshney U 2014 Mobile health: four emerging themes of research Decis. Support Syst. 66 20-35

[118] Wu F and Asada H 2014 Supernumerary robotic fingers: an alternative upper-limb prosthesis Dynamic Systems and Conf. vol 46193 (American Society of Mechanical Engineers) p V002T16A009

[119] Abras C et al 2004 User-centered design Bainbridge, W. Encyclopedia of Human-Computer Interaction vol 37 (Thousand Oaks: Sage Publications) pp 445-56

[120] Seitz J A 2000 The bodily basis of thought New Ideas Psychol. 18 23-40

[121] Faisal A, Stout D, Apel J and Bradley B 2010 The manipulative complexity of lower paleolithic stone toolmaking PLoS One 5 e 13718

[122] Harvey M, Langheinrich M and Ward G 2016 Remembering through lifelogging: a survey of human memory augmentation Pervasive Mobile Comput. 27 14-26

[123] Musk E and Neuralink 2019 An integrated brain-machine interface platform with thousands of channels J. Med. Internet Res. 21 e16194

[124] Santarnecchi E and Rossi S 2016 Advances in the neuroscience of intelligence: from brain connectivity to brain perturbation Spanish J. Psychol. 19

[125] Kandel E R, Schwartz J H, Jessell, T M, Siegelbaum S A and Hudspeth A 2013 Principles of Neural Science, 5th edn (McGraw-Hill Education: Principles of Neural Science)

[126] Noccaro A, Raiano L, Pinardi M, Formica D and Di Pino G 2020 A novel proprioceptive feedback system for supernumerary robotic limb 2020 8th IEEE RAS/EMBS Int. Conf. for Biomedical Robotics and Biomechatronics (BioRob) (IEEE) pp 1024-9

[127] Abdi E, Burdet E, Bouri M and Bleuler H 2015 Control of a supernumerary robotic hand by foot: an experimental study in virtual reality PLoS One 10 e 0134501

[128] Abdi E, Bouri M, Himidan S, Burdet E and Bleuler H 2016 Third arm manipulation for surgical applications: an experimental study New Trends in Medical and Service Robots (Berlin: Springer) pp 153-63 